\title{
Einstein-Cartan calculus for exceptional geometry
}

\author{
Hadi Godazgar, ${ }^{a}$ Mahdi Godazgar ${ }^{a}$ and Hermann Nicolai ${ }^{b}$ \\ ${ }^{a}$ DAMTP, Centre for Mathematical Sciences, University of Cambridge, \\ Wilberforce Road, Cambridge, CB3 0WA, U.K. \\ ${ }^{b}$ Max-Planck-Institut für Gravitationsphysik, Albert-Einstein-Institut, \\ Am Mühlenberg 1, D-14476 Potsdam, Germany \\ E-mail: H.M.Godazgar@damtp.cam.ac.uk, M.M.Godazgar@damtp.cam.ac.uk, \\ Hermann. Nicolai@aei.mpg.de
}

ABSTRACT: In this paper we establish and clarify the link between the recently found $\mathrm{E}_{7(7)}$ generalised geometric structures, which are based on the SU(8) invariant reformulation of $D=11$ supergravity proposed long ago, and newer results obtained in the framework of recent approaches to generalised geometry, where $\mathrm{E}_{7(7)}$ duality is built in and manifest from the outset. In making this connection, the so-called generalised vielbein postulate plays a key role. We explicitly show how this postulate can be used to define an $\mathrm{E}_{7(7)}$ valued affine connection and an associated covariant derivative, which yields a generalised curvature tensor for the $\mathrm{E}_{7(7)}$ based exceptional geometry. The analysis of the generalised vielbein postulate also provides a natural explanation for the emergence of the embedding tensor from higher dimensions.

KEywords: Differential and Algebraic Geometry, M-Theory, String Duality

ARXIV EPRINT: 1401.5984 


\section{Contents}

1 Introduction 1

2 Preliminaries $\quad 4$

2.1 Generalised vielbein 4

$\begin{array}{lll}2.2 \text { Vector fields } & 7\end{array}$

3 Generalised vielbein postulate $\quad 9$

4 Generalised diffeomorphisms $\quad 12$

5 Generalised vielbein postulates and generalised geometry 15

6 Generalised $\mathrm{E}_{7(7)}$ curvature $\quad 18$

$\begin{array}{lll}7 & \text { Discussion } & 21\end{array}$

$\begin{array}{lll}\text { A } & \mathbf{E}_{7(7)} \text { algebra and identities } & 22\end{array}$

$\begin{array}{ll}\text { B Generalised covariance of the curvature tensor } & 23\end{array}$

\section{Introduction}

Recent progress [1], along the lines of an older proposal [2], on understanding the extent to which the $\mathrm{E}_{7(7)}$ Cremmer-Julia duality symmetry $[3,4]$ is inherent to the full $D=11$ supergravity theory [5] has lead to a new formulation of the $D=11$ theory, which apart from pointing to new geometric structures in eleven dimensions, provides an appropriate framework in which to address questions regarding the relation between $D=11$ supergravity and four-dimensional maximal gauged supergravity theories $[6,7]$. In this paper, we will clarify the relation of these results to more recent approaches to generalised geometry, especially [8-10], and show how a synthesis of the different approaches emerges.

The formalism of ref. [1] is based on the SU(8) invariant reformulation of $D=11$ supergravity [2], in which the local and global gravitational symmetries of the elevendimensional theory are abandoned and one performs a $4+7$ split of all fields in the theory. Importantly, dependence on all eleven coordinates is retained throughout and one remains on-shell equivalent to the original theory throughout the construction. An essential characteristic of the analysis of ref. [2], and a main distinguishing feature in comparison with more recent work, is the use of supersymmetry transformations to find new $\mathrm{SU}(8)$ and $\mathrm{E}_{7(7)}$ structures in the eleven-dimensional theory. The most significant such structures are the "generalised vielbeine" $[1,2,11]$, which replace the eleven-dimensional fields that would 
contribute to scalar degrees of freedom in a reduction to four dimensions. As in [2], these are derived by considering the supersymmetry transformation of eleven-dimensional fields that would contribute to vector degrees of freedom in a reduction to four dimensions. A crucial ingredient in constructing the full set of "generalised vielbeine" is to consider dual fields in eleven dimensions. These building blocks are to be viewed as the components of a single $\mathrm{E}_{7(7)}$ 56-bein $\mathcal{V}$ that we shall henceforth simply refer to as the "generalised vielbein", in analogy with the terminology used in more recent literature [12]. In particular, the generalised vielbein as derived directly from the $D=11$ theory in [1] coincides with the generalised vielbein that lies at the heart of other recent approaches to generalised geometry [13] (see also [12]), where it is constructed from the $\mathrm{E}_{7(7)} / \mathrm{SU}(8)$ coset using an algebraic method known as non-linear realisation [14-16]. More recently, the generalised geometry ideas that have been used to describe the seven-dimensional sector of $D=11$ supergravity in a $4+7$ split have been extended to incorporate the four-dimensional part, in this way arriving at an $\mathrm{E}_{7(7)}$ covariant extension of the whole theory $[10,17]$.

An important aspect of the formalism developed in [1] is the fact that the components of the generalised vielbein satisfy differential constraints $[1,2]$ — called "generalised vielbein postulates" (GVPs) due to their resemblance to the usual vielbein postulate in differential geometry. It should be emphasised that here these equations are not postulated, but follow directly from the explicit expressions for the generalised vielbein in terms of the various fields and dual fields of $D=11$ supergravity. In this sense, the present approach is 'bottom up', in contrast to other approaches, where similar relations follow from more abstract geometrical reasoning. One of our main results here is to show how these ingredients can be used to develop an Einstein-Cartan calculus that is largely analogous to the one for the standard vielbein.

The GVPs divide into two sets: those in which the derivative acting on the component of the generalised vielbein is taken with respect to the $D=4$ directions and those in which the derivative is with respect to the $D=7$ directions. Using a terminology where "external" refers to $D=4$ in the $4+7$ split of $D=11$, and "internal" refers to $D=7$, even though we remain on-shell equivalent to the $D=11$ theory and no reduction is assumed, we refer to the former set as "external GVPs" and the latter set as "internal GVPs". The GVPs are important in establishing a link between the $D=11$ theory and $D=4$ maximal gauged theories derived as a reduction thereof. In particular, the external GVPs can be regarded as providing a higher dimensional origin of the embedding tensor [18-22], as has been explicitly demonstrated for the $S^{7}$ reduction [6] and ScherkSchwarz flux compactifications [7]. The relationship between $D=11$ supergravity and $D=4$ supergravity is an important aspect of the $\mathrm{SU}(8)$ invariant reformulation of the $D=11$ theory [2], and recent developments therefrom [1, 11], in, for example, establishing non-linear ansätze $[6,11,23,24]$ and consistency of the $S^{7}$ reduction $[25,26]$. Very recently, this aspect has also been studied in ref. [27] where the generalised vielbein is related by a generalised Scherk-Schwarz ansatz to the $\mathrm{E}_{d(d)}$ matrix parametrised by the scalars of maximal gauged supergravity. This allows them to verify/conjecture non-linear ansätze for various sphere reductions. The validity of the new ansätze can be established by an analysis along the lines of refs. $[2,6,11,23]$ for the appropriate sphere reductions. 
In this paper, we return to the reformulation of $D=11$ supergravity developed in [1] and proceed to make concrete the indications that there is an $\mathrm{E}_{7(7)}$ generalised geometry underlying the constructions there. In particular, we make contact with recent results in duality-manifest based approaches to generalised geometry $[8,9]^{1}$ that have focused on similar issues from a duality group perspective. We condense all the objects and equations, in particular the GVPs, into an $\mathrm{E}_{7(7)}$ covariant form such that the previous expressions can be obtained as particular components of the new expressions under $\mathrm{SL}(8)$ and $\mathrm{GL}(7)$ decompositions of $\mathrm{E}_{7(7)}$. Thus, even though general covariance in $D=11$ has been abandoned in the $4+7$ split, we obtain a reformulation that has general covariance in the $D=4$ directions and a "generalised general covariance" based on $\mathrm{E}_{7(7)}$ in the $D=7$ space in a manner consistent with the results of refs. [8-10].

A prerequisite for introducing $\mathrm{E}_{7(7)}$ covariance, and thus replacing $\mathrm{GL}(7)$ indices with $\mathrm{E}_{7(7)}$ indices, is that the seven-dimensional space on which the generalised geometry is constructed apparently requires an extension to a 56 -dimensional space ${ }^{2}$ such that the seven internal coordinates $\left\{y^{m}\right\}$ are extended to a set of 56 internal coordinates $\left\{y^{\mathcal{M}}\right\}$, where $\mathcal{M}$ labels the $\mathbf{5 6}$ representation of $\mathrm{E}_{7(7)}$ [13]. However, in order for the geometric structures, such as the algebra of generalised diffeomorphisms, to be consistent one must impose a constraint, the section condition, that ultimately reduces the enlarged space to an at most seven-dimensional space $[8,9]$. While the necessity of such a restriction is plainly evident from the fact that no consistent supergravity appears to exist beyond eleven dimensions, its necessity can also be seen from a more geometrical perspective: supposing that the generalised vielbein $\mathcal{V}$ did depend on 56 internal coordinates, we would have the textbook formula

$$
\mathcal{V}_{\mathcal{M}}(y)=\mathcal{V}_{\mathcal{N}}^{\prime}\left(y^{\prime}\right) \frac{\partial y^{\mathcal{N}}}{\partial y^{\mathcal{M}}}
$$

for the transformation under arbitrary diffeomorphisms in 56 dimensions. However, the transition matrix $\partial y^{\prime \mathcal{M}} / \partial y^{\mathcal{N}}$ being an element of GL(56), this operation would throw the 56-bein $\mathcal{V}$ out of the coset $\mathrm{E}_{7(7)} / \mathrm{SU}(8)$. One might therefore ask whether there exists a set of restricted diffeomorphisms in 56 dimensions, such that $\partial y^{\mathcal{M}} / \partial y^{\mathcal{N}} \in \mathrm{E}_{7(7)}$ and the transformed generalised vielbein remains in the coset. However, this possibility is excluded by Cartan's Theorem, according to which there do not exist 'exceptional algebras of vector fields' on manifolds, the only possibilities being (essentially) the algebras of ordinary diffeomorphisms, volume preserving diffeomorphisms and symplectomorphisms [36, 37] (see also ref. [38]). Similar comments apply to the $3+8$ split associated to the $\mathrm{E}_{8(8)}$ duality group, as already noted in [39]. In the context of $\mathrm{O}(d, d)$, the finite transformation law [40,41] is more general than the conventional transformation law given in equation (1.1) thereby allowing a transformation that is contained in $\mathrm{O}(d, d)$, but it is nevertheless necessary to have the section condition (see also refs. $[42,43]$ for global considerations).

\footnotetext{
${ }^{1}$ For further references see [28-35].

${ }^{2}$ More precisely, the 11-dimensional space-time manifold would have to be extended to a $(4+56)$ dimensional space, but we can ignore the dependence on the four external coordinates for the argument to be presented.
} 
In section 2, we review the required results from [1], rewriting them in a manner that makes their $\mathrm{E}_{7(7)}$ structure manifest. We rewrite the GVPs, in section 3, using the $\mathrm{E}_{7(7)}$ structures defined in section 2 . Then, in section 4 , we explicitly demonstrate how the coordinate and gauge transformations of the generalised vielbein can be packaged into a single transformation given by generalised diffeomorphisms [8]. Finally, in section 5, we similarly package the GVPs into single $\mathrm{E}_{7(7)}$ covariant equations. The equation corresponding to the external GVPs is precisely of the same form as the Cartan equation in four-dimensional maximal gauged theories, allowing us to identify the higher dimensional object, an operator, that gives the embedding tensor upon reduction to four dimensions. On the other hand, the internal GVP is the generalised geometric analogue of the vielbein postulate and yields the generalised connection for the generalised geometry. We give the transformation properties of the generalised connection. Furthermore, we find that a covariant derivative defined using the generalised connection transforms as a generalised tensor density of weight $1 / 2$ less than the weight of the generalised tensor on which the covariant derivative acts. Thus, a generalised Riemann curvature tensor obtained by commuting two covariant derivatives transforms as a generalised tensor density of weight -1 . We explicitly present the components of the generalised Riemann tensor and note that it is indeed generalised gauge covariant.

The conventions used in this paper are the same as those of ref. [2]. In particular, $M, N, \ldots$ and $A, B, \ldots$ denote eleven-dimensional spacetime and tangent space indices, respectively. Indices $A, B, \ldots$ are also used as $\mathrm{SU}(8)$ indices. However, it should be clear from the context what type of index is being referred to. Similarly, $\mu, \nu, \ldots$ and $\alpha, \beta, \ldots$, and $m, n, \ldots$ and $a, b, \ldots$ denote $D=4$ and $D=7$ spacetime and tangent space indices, respectively.

\section{Preliminaries}

\subsection{Generalised vielbein}

As explained in much detail in our previous work [1], a generalised vielbein $\mathcal{V}$, which can be viewed as a 56-bein of $\mathrm{E}_{7(7)}$, can be defined directly in eleven dimensions. This 56bein depends on the fields and on the dual fields of $D=11$ supergravity, as obtained by performing a $4+7$ split on the original fields, with all fields still depending on all eleven-dimensional coordinates. In particular, it depends on the siebenbein $e_{m}{ }^{a}$, which is obtained from a $4+7$ decomposition of the original elfbein of $D=11$ supergravity in a triangular gauge (which breaks the original tangent space symmetry $\mathrm{SO}(1,10)$ of the theory to $\mathrm{SO}(1,3) \times \mathrm{SO}(7))$ :

$$
E_{M}^{A}(x, y)=\left(\begin{array}{cc}
\Delta^{-1 / 2} e_{\mu}^{\alpha} & B_{\mu}{ }^{m} e_{m}^{a} \\
0 & e_{m}{ }^{a}
\end{array}\right), \quad \Delta \equiv \operatorname{det} e_{m}^{a} .
$$

Here, as usual, we split the eleven-dimensional coordinates $\left\{z^{M}\right\}$ into four external coordinates $\left\{x^{\mu}\right\}$ and seven internal coordinates $\left\{y^{m}\right\}$. The 3 -form and 6-form gauge fields, on 
which the 56-bein also depends, are linked via the duality relation

$$
\begin{aligned}
F_{M_{1} \cdots M_{7}}= & 7 ! D_{\left[M_{1}\right.} A_{\left.M_{2} \cdots M_{7}\right]}+7 ! \frac{\sqrt{2}}{2} A_{\left[M_{1} M_{2} M_{3}\right.} D_{M_{4}} A_{\left.M_{5} M_{6} M_{7}\right]} \\
& -\frac{\sqrt{2}}{192} i \epsilon_{M_{1} \cdots M_{11}}\left(\bar{\Psi}_{R} \tilde{\Gamma}^{M_{8} \cdots M_{11} R S} \Psi_{S}+12 \bar{\Psi}^{M_{8}} \tilde{\Gamma}^{M_{9} M_{10}} \Psi^{M_{11}}\right)
\end{aligned}
$$

in eleven dimensions, from which all pertinent relations linking the 4 -form and 7-form field strengths can be obtained by choosing the indices appropriately. Although we will ignore the fermionic terms in this duality relation in the remainder, it should be clear that this duality relation introduces a hidden dependence of the 56-bein (which we are about to present) on the fermionic fields as well.

A main result of [1] is thus the complete identification of the 56-bein in terms of the siebenbein, and the internal components of the 3 -form and the 6 -form, such that $\mathcal{V} \equiv \mathcal{V}\left(e, A^{(3)}, A^{(6)}\right)$. With proper $\mathrm{E}_{7(7)}$ normalisation, the components of the generalised vielbein are explicitly given by

$$
\begin{aligned}
& \mathcal{V}^{m}{ }_{A B}=-\frac{\sqrt{2}}{8} \Delta^{-1 / 2} \Gamma_{A B}^{m}, \\
& \mathcal{V}_{m n A B}=-\frac{\sqrt{2}}{8} \Delta^{-1 / 2}\left(\Gamma_{m n A B}+6 \sqrt{2} A_{m n p} \Gamma_{A B}^{p}\right), \\
& \mathcal{V}_{A B}^{m n}=-\frac{\sqrt{2}}{8} \cdot \frac{1}{5 !} \eta^{m n p_{1} \cdots p_{5}} \Delta^{-1 / 2}\left[\Gamma_{p_{1} \cdots p_{5} A B}+60 \sqrt{2} A_{p_{1} p_{2} p_{3}} \Gamma_{p_{4} p_{5} A B}\right. \\
& \left.-6 ! \sqrt{2}\left(A_{q p_{1} \cdots p_{5}}-\frac{\sqrt{2}}{4} A_{q p_{1} p_{2}} A_{p_{3} p_{4} p_{5}}\right) \Gamma_{A B}^{q}\right] \\
& \mathcal{V}_{m A B}=-\frac{\sqrt{2}}{8} \cdot \frac{1}{7 !} \eta^{p_{1} \cdots p_{7}} \Delta^{-1 / 2}\left[\left(\Gamma_{p_{1} \cdots p_{7}} \Gamma_{m}\right)_{A B}+126 \sqrt{2} A_{m p_{1} p_{2}} \Gamma_{p_{3} \cdots p_{7} A B}\right. \\
& +3 \sqrt{2} \times 7 !\left(A_{m p_{1} \cdots p_{5}}+\frac{\sqrt{2}}{4} A_{m p_{1} p_{2}} A_{p_{3} p_{4} p_{5}}\right) \Gamma_{p_{6} p_{7} A B} \\
& \left.+\frac{9 !}{2}\left(A_{m p_{1} \cdots p_{5}}+\frac{\sqrt{2}}{12} A_{m p_{1} p_{2}} A_{p_{3} p_{4} p_{5}}\right) A_{p_{6} p_{7} q} \Gamma_{A B}^{q}\right],
\end{aligned}
$$

where $\Gamma^{m} \equiv e^{m}{ }_{a} \Gamma^{a}$ are the $D=7$ gamma matrices with seven-dimensional curved indices and $\eta^{m_{1} \ldots m_{7}}$ is the seven-dimensional permutation symbol (tensor density of weight +1 ). These expressions are obtained by insisting on the $\mathrm{E}_{7(7)}$ covariance of the supersymmetry variations (after appropriate field redefinitions), see also remarks in section 2.2 below.

The vielbein is subject to local $\mathrm{SU}(8)$ rotations (depending on all eleven coordinates), such that the above expressions in terms of quantities of $D=11$ supergravity correspond to a special gauge choice, as explained already in [2]. Furthermore, complex conjugation raises (lowers) $\mathrm{SU}(8)$ indices

$$
\mathcal{V}_{\mathrm{MN}} A B \equiv\left(\mathcal{V}_{\mathrm{MN} A B}\right)^{*}, \quad \mathcal{V}^{\mathrm{MN} A B} \equiv\left(\mathcal{V}_{A B}^{\mathrm{MN}}\right)^{*},
$$

where we have combined the GL(7) indices $m, n, \ldots$ into $\mathrm{SL}(8)$ indices $\mathrm{M}, \mathrm{N}, \ldots$ according to ${ }^{3}$

$$
\mathcal{V}_{\mathrm{MN}} \equiv\left(\mathcal{V}_{m n}, \mathcal{V}_{m 8}\right), \quad \mathcal{V}^{\mathrm{MN}} \equiv\left(\mathcal{V}^{m n}, \mathcal{V}^{m 8}\right)
$$

\footnotetext{
${ }^{3}$ For brevity, we will often use the simplifying notation $\mathcal{V}^{m} \equiv \mathcal{V}^{m 8}=-\mathcal{V}^{8 m}$ and $\mathcal{B}^{m} \equiv \mathcal{B}^{m 8}=-\mathcal{B}^{8 m}$, etc.
} 
That is, complex conjugation only affects the $\mathrm{SU}(8)$ indices. We will also use proper $\mathrm{E}_{7(7)}$ indices $\mathcal{M}, \mathcal{N}, \ldots$ corresponding to the $\mathbf{5 6}$ representation, such that

$$
\mathcal{V}_{\mathcal{M}} \equiv\left(\mathcal{V}_{\mathrm{MN}}, \mathcal{V}^{\mathrm{MN}}\right), \quad \mathcal{V}^{\mathcal{M}}=\Omega^{\mathcal{M N}} \mathcal{V}_{\mathcal{N}} \equiv\left(\mathcal{V}^{\mathrm{MN}},-\mathcal{V}_{\mathrm{MN}}\right)
$$

where the components of the symplectic form $\Omega^{\mathcal{M N}}$ are

$$
\begin{array}{ll}
\Omega^{\mathrm{MN}}{ }_{\mathrm{PQ}}=\delta_{\mathrm{PQ}}^{\mathrm{MN}}, & \Omega_{\mathrm{MN}}^{\mathrm{PQ}}=-\delta_{\mathrm{MN}}^{\mathrm{PQ}}, \\
\Omega_{\mathrm{MN} \mathrm{PQ}}=0, & \Omega^{\mathrm{MN} \mathrm{PQ}}=0,
\end{array}
$$

and $\Omega_{\mathcal{M N}}$ is given by $\Omega^{\mathcal{M P}} \Omega_{\mathcal{N P}}=\delta_{\mathcal{N}}^{\mathcal{M}}$. Moreover, with the above normalisation, $\mathcal{V}$ satisfies the $\mathrm{E}_{7(7)}$ properties

$$
\begin{aligned}
\mathcal{V}_{\mathcal{M}}{ }^{A B} \mathcal{V}_{\mathcal{N} A B}-\mathcal{V}_{\mathcal{M} A B} \mathcal{V}_{\mathcal{N}}{ }^{A B} & =i \Omega_{\mathcal{M N}} \\
\Omega^{\mathcal{M N}} \mathcal{V}_{\mathcal{M}}{ }^{A B} \mathcal{V}_{\mathcal{N} C D} & =i \delta_{C D}^{A B} \\
\Omega^{\mathcal{M N}} \mathcal{V}_{\mathcal{M}}{ }^{A B} \mathcal{V}_{\mathcal{N}}{ }^{C D} & =0
\end{aligned}
$$

which can be directly verified from definitions (2.3)-(2.6). The generalised vielbein also satisfies the following $\mathrm{E}_{7(7)}$ covariant supersymmetry transformation

$$
\delta \mathcal{V}_{\mathcal{M A B}}=\sqrt{2} \Sigma_{A B C D} \mathcal{V}_{\mathcal{M}}{ }^{C D}
$$

with the complex self-dual SU(8) tensor

$$
\Sigma_{A B C D}=\bar{\varepsilon}_{[A} \chi_{B C D]}+\frac{1}{4 !} \epsilon_{A B C D E F G H} \bar{\varepsilon}^{E} \chi^{F G H} .
$$

In the $\mathrm{SU}(8)$ invariant reformulation, the $D=11$ gravitino $\Psi_{M}$ is rewritten in terms of $\mathrm{SU}(8)$ covariant chiral fermions $\varphi_{\mu}{ }^{A}$ and $\chi_{A B C}$ and their complex conjugates $\varphi_{\mu A}, \chi^{A B C}[2$, 4]. The precise relation between (2.12) and the $D=11$ supersymmetry variations also involves an $\mathrm{SU}(8)$ rotation that we have dropped.

In addition to local $\mathrm{SU}(8)$ transformations, the generalised vielbein is subject to several gauge transformations which it inherits from the fields on which it depends, to wit, internal diffeomorphisms, and the tensor gauge transformations associated to the 3 -form and the 6 -form gauge potentials. Recall that in our scheme all transformation parameters depend on eleven coordinates. The transformations under internal diffeomorphisms are straightforward to obtain:

$$
\begin{gathered}
\delta \mathcal{V}_{A B}^{m}=\xi^{p} \partial_{p} \mathcal{V}_{A B}^{m}-\partial_{p} \xi^{m} \mathcal{V}_{A B}^{p}-\frac{1}{2} \partial_{p} \xi^{p} \mathcal{V}_{A B}^{m}, \\
\delta \mathcal{V}_{m n A B}=\xi^{p} \partial_{p} \mathcal{V}_{m n A B}-2 \partial_{[m} \xi^{p} \mathcal{V}_{n] p}-\frac{1}{2} \partial_{p} \xi^{p} \mathcal{V}_{m n A B}, \\
\delta \mathcal{V}_{A B}^{m n}=\xi^{p} \partial_{p} \mathcal{V}_{A B}^{m n}+2 \partial_{p} \xi^{[m} \mathcal{V}_{A B}^{n] p}+\frac{1}{2} \partial_{p} \xi^{p} \mathcal{V}_{A B}^{m n}, \\
\delta \mathcal{V}_{m A B}=\xi^{p} \partial_{p} \mathcal{V}_{m A B}+\partial_{m} \xi^{p} \mathcal{V}_{p A B}+\frac{1}{2} \partial_{p} \xi^{p} \mathcal{V}_{m A B}
\end{gathered}
$$


Note that the density terms come from the overall factor of $\Delta^{ \pm 1 / 2}$ in the definition of $\mathcal{V}_{\mathcal{M}}$. With respect to the tensor gauge transformations, we have

$$
\delta A_{m n p}=3 ! \partial_{[m} \xi_{n p]}, \quad \delta A_{m n p q r s}=3 \sqrt{2} \partial_{[m} \xi_{n p} A_{q r s]}
$$

and

$$
\delta A_{m n p}=0, \quad \delta A_{m n p q r s}=6 ! \partial_{[m} \xi_{n p q r s]}
$$

with the 2-form and 5-form gauge parameters $\xi_{m n}$ and $\xi_{m n p q r}$, respectively. Substituting these transformations into the explicit expressions for the generalised vielbein components in (2.3)-(2.6), it is straightforward to deduce the transformation properties

$$
\begin{aligned}
\delta \mathcal{V}_{A B}^{m} & =0, & \delta \mathcal{V}_{m n A B} & =36 \sqrt{2} \partial_{[m} \xi_{n p]} \mathcal{V}_{A B}^{p}, \\
\delta \mathcal{V}^{m n}{ }_{A B} & =3 \sqrt{2} \eta^{\text {mnpqrst }} \partial_{p} \xi_{q r} \mathcal{V}_{s t A B}, & \delta \mathcal{V}_{m A B} & =18 \sqrt{2} \partial_{[m} \xi_{n p]} \mathcal{V}_{A B}^{n p},
\end{aligned}
$$

and

$$
\begin{aligned}
& \delta \mathcal{V}_{A B}^{m}=\delta \mathcal{V}_{m n A B}=0, \quad \delta \mathcal{V}_{A B}^{m n}=-6 \cdot 6 ! \sqrt{2} \eta^{m n p_{1} \cdots p_{5}} \partial_{[q} \xi_{\left.p_{1} \cdots p_{5}\right]} \mathcal{V}_{A B}^{q} \\
& \delta \mathcal{V}_{m A B}=3 \cdot 6 ! \sqrt{2} \eta^{n_{1} \cdots n_{7}} \partial_{[m} \xi_{\left.n_{1} \cdots n_{5}\right]} \mathcal{V}_{n_{6} n_{7} A B}
\end{aligned}
$$

We already see here that these transformation parameters can be nicely combined as

$$
\Lambda^{\mathcal{M}} \equiv\left(\Lambda^{m}, \Lambda_{m n}, \Lambda^{m n}, 0\right)
$$

where $\Lambda^{m} \sim \xi^{m}, \Lambda_{m n} \sim \xi_{m n}$ and $\Lambda^{m n} \sim \eta^{m n p_{1} \cdots p_{5}} \xi_{p_{1} \cdots p_{5}}$ (the precise coefficients will be conveniently chosen later). In this way ordinary diffeomorphisms and tensor gauge transformations are unified into a single set of transformations. This will be shown explicitly in section 4, where we will consider generalised diffeomorphisms and show how the above transformations can be compactly written in terms of a single generalised Lie derivative, see equation (4.2). The 'missing' seven components $\Lambda_{m}$ in this identification are obviously associated with 'dual' internal diffeomorphisms, but will actually be seen to drop out.

\subsection{Vector fields}

The components of the generalised vielbein can be obtained by considering the supersymmetry of a set of eleven-dimensional fields with one $D=4$ index $[1,2,11]$. As such they are known as vectors in accord with the convention of using four-dimensional language for analogous $D=11$ structures adopted here. We similarly combine the vectors into a $\mathbf{5 6}$ of $\mathrm{E}_{7(7)}$

$$
\mathcal{B}_{\mu}^{\mathcal{M}}=\left(\mathcal{B}_{\mu}^{\mathrm{MN}}, \mathcal{B}_{\mu \mathrm{MN}}\right)
$$


The proper definitions of these 56 vector fields follow from the identifications

$$
\begin{aligned}
\mathcal{B}_{\mu}{ }^{m}= & -\frac{1}{2} B_{\mu}{ }^{m}, \quad \mathcal{B}_{\mu m n}=-3 \sqrt{2}\left(A_{\mu m n}-B_{\mu}{ }^{p} A_{p m n}\right), \\
\mathcal{B}_{\mu}{ }^{m n}= & -3 \sqrt{2} \eta^{m n p_{1} \ldots p_{5}}\left(A_{\mu p_{1} \cdots p_{5}}-B_{\mu}{ }^{q} A_{q p_{1} \cdots p_{5}}-\frac{\sqrt{2}}{4}\left(A_{\mu p_{1} p_{2}}-B_{\mu}^{q} A_{q p_{1} p_{2}}\right) A_{p_{3} p_{4} p_{5}}\right) \\
\mathcal{B}_{\mu m}= & -18 \eta^{n_{1} \ldots n_{7}}\left(A_{\mu n_{1} \ldots n_{7}, m}+(3 \tilde{c}-1)\left(A_{\mu n_{1} \ldots n_{5}}-B_{\mu}^{p} A_{p n_{1} \ldots n_{5}}\right) A_{n_{6} n_{7} m}\right. \\
& \left.+\tilde{c} A_{n_{1} \ldots n_{6}}\left(A_{\mu n_{7} m}-B_{\mu}^{p} A_{p n_{7} m}\right)+\frac{\sqrt{2}}{12}\left(A_{\mu n_{1} n_{2}}-B_{\mu}^{p} A_{p n_{1} n_{2}}\right) A_{n_{3} n_{4} n_{5}} A_{n_{6} n_{7} m}\right),
\end{aligned}
$$

where $\tilde{c}$ is an undetermined constant. These are related to the generalised vielbein via the following supersymmetry transformation $[1,2,11]$

$$
\delta \mathcal{B}_{\mu}^{\mathcal{M}}=i \Omega^{\mathcal{M N}} \mathcal{V}_{\mathcal{N} A B}\left(2 \sqrt{2} \bar{\varepsilon}^{A} \varphi_{\mu}^{B}+\bar{\varepsilon}_{C} \gamma_{\mu} \chi^{A B C}\right)+\text { h.c. }
$$

using the supersymmetry transformations of the fields given in $[1,2,11]$. In particular [1]

$$
\begin{aligned}
\delta A_{\mu m_{1} \ldots m_{7}, n}= & -\frac{1}{9 !}\left(\bar{\varepsilon} \tilde{\Gamma}_{\mu m_{1} \ldots m_{7}} \Psi_{n}-8 \bar{\varepsilon} \tilde{\Gamma}_{n} \tilde{\Gamma}_{\left[\mu m_{1} \ldots m_{6}\right.} \Psi_{\left.m_{7}\right]}\right)+\frac{\sqrt{2} \tilde{c}}{5 !} \bar{\varepsilon} \tilde{\Gamma}_{\left[\mu m_{1} \ldots m_{4}\right.} \Psi_{m_{5}} A_{\left.m_{6} m_{7}\right] n} \\
& +\frac{\sqrt{2}}{3} \bar{\varepsilon} \tilde{\Gamma}_{\left[\mu m_{1}\right.} \Psi_{m_{2}}\left(A_{\left.m_{3} \ldots m_{7}\right] n}+\frac{\sqrt{2}}{12} A_{m_{3} \ldots m_{5}} A_{\left.m_{6} m_{7}\right] n}\right) \\
& -\sqrt{2} \tilde{c} \bar{\varepsilon} \tilde{\Gamma}_{\left[\mu m_{1}\right.} \Psi_{m_{2}}\left(A_{\left.m_{3} \ldots m_{7}\right] n}+\frac{\sqrt{2}}{4} A_{m_{3} \ldots m_{5}} A_{\left.m_{6} m_{7}\right] n}\right)
\end{aligned}
$$

where $\Psi_{m}$ is the component of the $D=11$ gravitino along the internal directions (prior to any redefinition).

The transformation of the components of $\mathcal{B}_{\mu}^{\mathcal{M}}$ under internal diffeomorphisms is

$$
\begin{aligned}
\delta \mathcal{B}_{\mu}{ }^{m} & =\xi^{p} \partial_{p} \mathcal{B}_{\mu}{ }^{m}-\partial_{p} \xi^{m} \mathcal{B}_{\mu}{ }^{p} \\
\delta \mathcal{B}_{\mu m n} & =\xi^{p} \partial_{p} \mathcal{B}_{\mu m n}-2 \partial_{[m} \xi^{p} \mathcal{B}_{\mu n] p}, \\
\delta \mathcal{B}_{\mu}{ }^{m n} & =\xi^{p} \partial_{p} \mathcal{B}_{\mu}{ }^{m n}+2 \partial_{p} \xi^{[m} \mathcal{B}_{\mu}{ }^{n] p}+\partial_{p} \xi^{p} \mathcal{B}_{\mu}{ }^{m n}
\end{aligned}
$$

We note that $\mathcal{B}_{\mu}{ }^{m n}$ transforms as a tensor density of weight 1 because of the tensor density $\eta$ in its definition, (2.21). The transformation of $\mathcal{B}_{\mu}^{\mathcal{M}}$ under internal 2 -form and 5 -form gauge transformations is

$$
\begin{aligned}
\delta \mathcal{B}_{\mu}{ }^{m} & =0, \quad \delta \mathcal{B}_{\mu m n}=-36 \sqrt{2} \partial_{[m} \xi_{n p]} \mathcal{B}_{\mu}{ }^{p} \\
\delta \mathcal{B}_{\mu}{ }^{m n} & =-3 \sqrt{2} \Delta \epsilon^{m n p_{1} \cdots p_{5}} \partial_{p_{1}} \xi_{p_{2} p_{3}} \mathcal{B}_{\mu p_{4} p_{5}} .
\end{aligned}
$$

and

$$
\delta \mathcal{B}_{\mu}{ }^{m}=\delta \mathcal{B}_{\mu m n}=0, \quad \delta \mathcal{B}_{\mu}{ }^{m n}=-6 \cdot 6 ! \sqrt{2} \eta^{m n p_{1} \cdots p_{5}} \partial_{[q} \xi_{\left.p_{1} \cdots p_{5}\right]} \mathcal{B}_{\mu}{ }^{q}
$$


Since we do not know at this point how $A_{\mu m_{1} \ldots m_{7}, n}$ transforms under coordinate, 2 -form and 5-form gauge transformation we cannot, yet, determine the gauge transformation rule for the final component $\mathcal{B}_{\mu m}$. Let us nevertheless anticipate the results of section 4 , where we will find the transformation rule from the $\mathrm{E}_{7(7)}$ structure of internal coordinate and gauge transformations:

$$
\begin{aligned}
\delta \mathcal{B}_{\mu m} & =\xi^{p} \partial_{p} \mathcal{B}_{\mu m}+\partial_{m} \xi^{p} \mathcal{B}_{\mu p}+\partial_{p} \xi^{p} \mathcal{B}_{\mu m}, \\
\delta \mathcal{B}_{\mu m} & =-18 \sqrt{2} \partial_{[m} \xi_{p q]} \mathcal{B}_{\mu}{ }^{p q}, \quad \delta \mathcal{B}_{\mu m}=3 \cdot 6 ! \sqrt{2} \eta^{n_{1} \cdots n_{7}} \partial_{[m} \xi_{\left.n_{1} \cdots n_{5}\right]} \mathcal{B}_{\mu n_{6} n_{7}}
\end{aligned}
$$

for coordinate, 2-form and 5-form gauge transformations, respectively. Going backwards from these expressions, we can deduce that $A_{\mu n_{1} \ldots n_{7}, m}$ transforms as a tensor under internal coordinate transformations and under 2-form and 5-form gauge transformations it transforms as:

$$
\begin{aligned}
\delta A_{\mu n_{1} \ldots n_{7}, m}= & -18 \tilde{c} \partial_{[m} \xi_{\left.n_{1} n_{2}\right]} A_{\mu n_{3} \ldots n_{7}}+\sqrt{2}(9 \tilde{c}-2) \partial_{n_{1}} \xi_{n_{2} n_{3}} A_{\mu n_{4} n_{5}} A_{m n_{6} n_{7}} \\
& -\frac{(9 \tilde{c}-2)}{\sqrt{2}} \partial_{[m} \xi_{\left.n_{1} n_{2}\right]} A_{\mu n_{3} n_{4}} A_{n_{5} \ldots n_{7}}, \\
\delta A_{\mu n_{1} \ldots n_{7}, m}= & -6 !(3 \tilde{c}-1) \partial_{[m} \xi_{\left.n_{1} \ldots n_{5}\right]} A_{\mu n_{6} n_{7}},
\end{aligned}
$$

respectively. Here $\tilde{c}$ is the undetermined constant that appeared already in [1], and that is also not fixed by imposing $\mathrm{E}_{7(7)}$ covariance. As for the generalised vielbein, we will show that the formulae $(2.25),(2.26)$ and $(2.28)$, together with the action of internal diffeomorphisms, can be compactly assembled into a single $\mathrm{E}_{7(7)}$ covariant formula, (4.12).

\section{Generalised vielbein postulate}

The generalised vielbeine satisfy differential constraints along the four external and the seven internal directions, which are called generalised vielbeine postulates (GVPs) in analogy with the usual vielbein postulate in differential geometry. These constraints are identities that can be directly verified from the explicit expressions given above, just like the usual vielbein postulate is an identity when the affine connection and the spin connection are expressed in terms of the usual vielbein.

The external GVPs, which are the GVPs along the $d=4$ directions are of the form ${ }^{4}$

$$
\begin{gathered}
\partial_{\mu} \mathcal{V}^{m}{ }_{A B}+\mathcal{Q}_{\mu[A}^{C} \mathcal{V}^{m}{ }_{B] C}+2 \mathcal{B}_{\mu}{ }^{n} D_{n} \mathcal{V}^{m}{ }_{A B}-2 D_{n} \mathcal{B}_{\mu}{ }^{m} \mathcal{V}_{A B}-D_{n} \mathcal{B}_{\mu}{ }^{n} \mathcal{V}_{A B}=\mathcal{P}_{\mu A B C D} \mathcal{V}^{m C D} \\
\partial_{\mu} \mathcal{V}_{m n A B}+\mathcal{Q}_{\mu[A}^{C} \mathcal{V}_{|m n| B] C}+2 \mathcal{B}_{\mu}{ }^{p} D_{p} \mathcal{V}_{m n A B}-4 D_{[m} \mathcal{B}_{|\mu|}{ }^{p} \mathcal{V}_{n] p A B} \\
-D_{p} \mathcal{B}_{\mu}{ }^{p} \mathcal{V}_{m n A B}+6 D_{[m} \mathcal{B}_{|\mu| n p]} \mathcal{V}_{A B}=\mathcal{P}_{\mu A B C D} \mathcal{V}_{m n}{ }^{C D}
\end{gathered}
$$

${ }^{4}$ Note that the sign in front of the $\mathcal{P}$ structures in both the external and internal GVPs is opposite to what appears in the GVPs as written in ref. [1]. This is because of a differing definition of the generalised vielbein $\mathcal{V}$ - more specifically, an extra factor of $i$ in the definition of $\mathcal{V}$. 


$$
\begin{gathered}
\partial_{\mu} \mathcal{V}_{A B}^{m n}+\mathcal{Q}_{\mu[A}^{C} \mathcal{V}^{m n}{ }_{B] C}+2 \mathcal{B}_{\mu}{ }^{p} D_{p} \mathcal{V}_{A B}^{m n}+6 D_{p} \mathcal{B}_{\mu}{ }^{[m} \mathcal{V}_{A B}^{n p]} \\
-D_{p} \mathcal{B}_{\mu}{ }^{p} \mathcal{V}_{A B}^{m n}+\frac{1}{2} \eta^{m n p_{1} \ldots p_{5}} D_{p_{1}} \mathcal{B}_{\mu p_{2} p_{3}} \mathcal{V}_{p_{4} p_{5}}{ }_{A B}+4 D_{p} \mathcal{B}_{\mu}{ }^{p[m} \mathcal{V}_{A B}^{n]}=\mathcal{P}_{\mu A B C D} \mathcal{V}^{m n C D} \\
\partial_{\mu} \mathcal{V}_{m A B}+\mathcal{Q}_{\mu[A}^{C} \mathcal{V}_{m B] C}+2 \mathcal{B}_{\mu}{ }^{p} D_{p} \mathcal{V}_{m A B}+2 D_{m} \mathcal{B}_{\mu}{ }^{p} \mathcal{V}_{p A B} \\
\quad+D_{p} \mathcal{B}_{\mu}{ }^{p} \mathcal{V}_{m A B}+3 D_{[m} \mathcal{B}_{|\mu| p q]} \mathcal{V}_{A B}^{p q}-2 D_{p} \mathcal{B}_{\mu}{ }^{p q} \mathcal{V}_{q m A B}=\mathcal{P}_{\mu A B C D} \mathcal{V}_{m}{ }^{C D}
\end{gathered}
$$

where $D_{m}$ is the covariant derivative with respect to seven-dimensional diffeomorphisms, e.g.

$$
D_{m} \mathcal{B}_{\mu}{ }^{n} \equiv \partial_{m} \mathcal{B}_{\mu}{ }^{n}+\Gamma_{m p}^{n} \mathcal{B}_{\mu}{ }^{p}
$$

with the internal affine connection $\Gamma_{m n}^{p}$. In GVPs, above, the combination of components of the vector field $\mathcal{B}_{\mu}{ }^{\mathcal{M}}$ and the generalised vielbein in each term is exactly such that the discrepancy in the weights of the components of the generalised vielbein is compensated by the differing weights in the components of the vector field $\mathcal{B}_{\mu}{ }^{\mathcal{M}}$. Hence the weights of the terms in each GVP are consistent.

Note that in previous work $[1,2]$ these relations were given without the affine connection terms, but the relations above are still equivalent to the original ones (see [44]), as all terms containing the affine connections cancel in the above relations, as well as the ones given below. The connection coefficients are of the form

$$
\begin{aligned}
\mathcal{Q}_{\mu B}^{A}= & -\frac{1}{2}\left[e^{m}{ }_{a} D_{m} B_{\mu}{ }^{n} e_{n b}-\left(e^{p}{ }_{a} \mathcal{D}_{\mu} e_{p b}\right)\right] \Gamma_{A B}^{a b}-\frac{\sqrt{2}}{12} e_{\mu}{ }^{\alpha}\left(F_{\alpha a b c} \Gamma_{A B}^{a b c}-\eta_{\alpha \beta \gamma \delta} F^{\beta \gamma \delta a} \Gamma_{a A B}\right), \\
\mathcal{P}_{\mu A B C D}= & \frac{3}{4}\left[e^{m}{ }_{a} D_{m} B_{\mu}{ }^{n} e_{n b}-\left(e^{p}{ }_{a} \mathcal{D}_{\mu} e_{p b}\right)\right] \Gamma_{[A B}^{a} \Gamma_{C D]}^{b}-\frac{\sqrt{2}}{8} e_{\mu}{ }^{\alpha} F_{a b c \alpha} \Gamma_{[A B}^{a} \Gamma_{C D]}^{b c} \\
& -\frac{\sqrt{2}}{48} e_{\mu \alpha} \eta^{\alpha \beta \gamma \delta} F_{a \beta \gamma \delta} \Gamma_{b[A B} \Gamma_{C D]}^{a b},
\end{aligned}
$$

where

$$
\mathcal{D}_{\mu} \equiv \partial_{\mu}-B_{\mu}{ }^{m} D_{m} \equiv \partial_{\mu}+2 \mathcal{B}_{\mu}{ }^{m} D_{m} .
$$

In the dimensionally reduced theory, the kinetic term for the scalar fields is $\propto$ $\mathcal{P}_{\mu}^{A B C D} \mathcal{P}_{A B C D}^{\mu}$, while the 'composite' $\mathrm{SU}(8)$ connection $\mathcal{Q}_{\mu}{ }^{A} B$ is required for the covariantisation of the fermionic couplings.

Similarly, the generalised vielbein satisfies a GVP along the internal directions. The relevant relations were derived in [1] and read

$$
\begin{gathered}
\partial_{p} \mathcal{V}_{A B}^{m}+\Gamma_{p n}^{m} \mathcal{V}_{A B}^{n}+\frac{1}{2} \Gamma_{p n}^{n} \mathcal{V}_{A B}^{m}+\mathcal{Q}_{p[A}^{C} \mathcal{V}_{B] C}^{m}=\mathcal{P}_{p A B C D} \mathcal{V}^{m C D} \\
\partial_{p} \mathcal{V}_{m n A B}+2 \Gamma_{p[m}^{q} \mathcal{V}_{n] q} A B+\frac{1}{2} \Gamma_{p q}^{q} \mathcal{V}_{m n A B}-6 \sqrt{2} \Xi_{p \mid m n q} \mathcal{V}_{A B}^{q} \\
+\mathcal{Q}_{p[A}^{C} \mathcal{V}_{m n B] C}=\mathcal{P}_{p A B C D} \mathcal{V}_{m n}{ }^{C D}, \\
\partial_{p} \mathcal{V}_{A B}^{m n}-2 \Gamma_{p q}^{[m} \mathcal{V}^{n] q}{ }_{A B}-\frac{1}{2} \Gamma_{p q}^{q} \mathcal{V}_{A B}^{m n}-6 \sqrt{2} \eta^{m n q_{1} \cdots q_{5}} \Xi_{p \mid q_{1} \ldots q_{6}} \mathcal{V}_{A B}^{q_{6}}{ }_{A B} \\
-\frac{1}{\sqrt{2}} \eta^{m n q_{1} \cdots q_{5}} \Xi_{p \mid q_{1} q_{2} q_{3}} \mathcal{V}_{q_{4} q_{5} A B}+\mathcal{Q}_{p[A}^{C} \mathcal{V}_{B] C}^{m n}=\mathcal{P}_{p A B C D} \mathcal{V}^{m n C D},
\end{gathered}
$$




$$
\begin{array}{r}
\partial_{p} \mathcal{V}_{m A B}-\Gamma_{p m}^{n} \mathcal{V}_{n A B}-\frac{1}{2} \Gamma_{p q}^{q} \mathcal{V}_{m A B}-\sqrt{2} \eta^{n_{1} \cdots n_{7}} \Xi_{p \mid n_{1} \cdots n_{6}} \mathcal{V}_{n_{7} m A B} \\
-3 \sqrt{2} \Xi_{p \mid r s m} \mathcal{V}^{r s}{ }_{A B}+\mathcal{Q}_{p[A}^{C} \mathcal{V}_{m B] C}=\mathcal{P}_{p A B C D} \mathcal{V}_{m}{ }^{C D}
\end{array}
$$

where the first few terms in each of the above equations correspond to the general covariant derivative, i.e.

$$
\begin{aligned}
D_{m} \mathcal{V}_{A B}^{n} & \equiv \partial_{m} \mathcal{V}_{A B}^{n}+\Gamma_{m p}^{n} \mathcal{V}_{A B}^{p}+\frac{1}{2} \Gamma_{m p}^{p} \mathcal{V}_{A B}^{n} \\
D_{p} \mathcal{V}_{m n A B} & \equiv \partial_{p} \mathcal{V}_{m n A B}+2 \Gamma_{p[m}^{q} \mathcal{V}_{n] q A B}+\frac{1}{2} \Gamma_{m p}^{p} \mathcal{V}_{A B}^{n}
\end{aligned}
$$

and so on. Note that the components of the generalised vielbein are densities with respect to internal coordinate transformations, hence the extra terms involving $\Gamma_{m n}^{n}$. Furthermore, the connection coefficients $\mathcal{Q}_{m B}^{A}$ and $\mathcal{P}_{m A B C D}$ are

$$
\begin{aligned}
& \mathcal{Q}_{m B}^{A}=-\frac{1}{2} \omega_{m a b} \Gamma_{A B}^{a b}+\frac{\sqrt{2}}{14} i f e_{m a} \Gamma_{A B}^{a}-\frac{\sqrt{2}}{48} e_{m}{ }^{a} F_{a b c d} \Gamma_{A B}^{b c d}, \\
& \mathcal{P}_{m A B C D}=\frac{\sqrt{2}}{56} \text { if } e_{m}{ }^{a} \Gamma_{a b[A B} \Gamma_{C D]}^{b}+\frac{\sqrt{2}}{32} e_{m}{ }^{a} F_{a b c d} \Gamma_{[A B}^{b} \Gamma_{C D]}^{c d},
\end{aligned}
$$

where

$$
f=-\frac{1}{24} i \eta^{\alpha \beta \gamma \delta} F_{\alpha \beta \gamma \delta}=-\frac{1}{7 !} \eta^{a_{1} \ldots a_{7}} F_{a_{1} \ldots a_{7}}
$$

The above connection coefficients can also be written in a more suggestive form

$$
\begin{aligned}
\mathcal{Q}_{m B}^{A} & =-\frac{1}{2} \omega_{m a b} \Gamma_{A B}^{a b}+\frac{\sqrt{2}}{14 \cdot 6 !} F_{m a_{1} \ldots a_{6}} \Gamma_{A B}^{a_{1} \ldots a_{6}}-\frac{\sqrt{2}}{48} F_{m a b c} \Gamma_{A B}^{a b c}, \\
\mathcal{P}_{m A B C D} & =-\frac{\sqrt{2}}{56 \cdot 5 !} F_{m a_{1} \ldots a_{6}} \Gamma_{[A B}^{a_{1}} \Gamma_{C D]}^{a_{2} \ldots a_{6}}+\frac{\sqrt{2}}{32} F_{m a b c} \Gamma_{[A B}^{a} \Gamma_{C D]}^{b c},
\end{aligned}
$$

whence it is clear that they are invariant under 2-form and 5-form gauge transformations. The expressions for $\mathcal{Q}_{m B}^{A}$ and $\mathcal{P}_{m A B C D}$ given here differ from the expressions given before ${ }^{5}$ because of the replacement

$$
e^{p}{ }_{a} \partial_{m} e_{p b} \rightarrow e^{p}{ }_{a} D_{m} e_{p b} \equiv-\omega_{m a b}
$$

where $D_{m} e_{n a} \equiv \partial_{m} e_{n a}-\Gamma_{m n}^{p} e_{p a}$, so that $\omega_{m a b}$ is just the usual spin connection (these modifications to the GVP were already introduced in [44]). By contrast there is now no contribution to $\mathcal{P}_{m A B C D}$ from the derivative of the siebenbein because the spin connection is antisymmetric in $[a b]$ and thus vanishes when contracted with $\Gamma_{[A B}^{a} \Gamma_{C D]}^{b}$. Nevertheless, the GVPs are fully equivalent to the ones given previously, with the only difference being that some of the terms have now been absorbed into the affine connection terms. One advantage of this rearrangement is that both $\mathcal{Q}_{m}$ and $\mathcal{P}_{m}$ now transform as proper vectors under internal diffeomorphisms, unlike the expressions originally given in [2].

\footnotetext{
${ }^{5}$ See equations (3.33) and (3.34) of ref. [2].
} 
The essential new feature in the internal GVPs (3.9)-(3.15) is the appearance of new affine connection coefficients associated with the form fields, to wit,

$$
\begin{aligned}
\Xi_{p \mid m n q} \equiv & D_{p} A_{m n q}-\frac{1}{4 !} F_{p m n q}, \\
\Xi_{p \mid m_{1} \cdots m_{6}} \equiv & D_{p} A_{m_{1} \cdots m_{6}}+\frac{\sqrt{2}}{48} F_{p\left[m_{1} m_{2} m_{3}\right.} A_{\left.m_{4} m_{5} m_{6}\right]} \\
& \quad-\frac{\sqrt{2}}{2}\left(D_{p} A_{\left[m_{1} m_{2} m_{3}\right.}-\frac{1}{4 !} F_{p\left[m_{1} m_{2} m_{3}\right.}\right) A_{\left.m_{4} m_{5} m_{6}\right]}-\frac{1}{7 !} F_{p m_{1} \ldots m_{6}} .
\end{aligned}
$$

Observe that the above expressions vanish upon full antisymmetrisation:

$$
\Xi_{[p \mid m n q]}=0, \quad \Xi_{\left[p \mid m_{1} \cdots m_{6}\right]}=0
$$

so that the gauge invariant 4-form and 7-form field strengths are uniformly projected out.

Under 2-form and 5-form gauge transformations, respectively, the connections transform as

$$
\begin{aligned}
\delta \Xi_{p \mid m n q} & =3 ! D_{p} D_{[m} \xi_{n q]} \\
\delta \Xi_{p \mid m_{1} \cdots m_{6}} & =-3 ! \sqrt{2}\left(D_{p} A_{\left[m_{1} m_{2} m_{3}\right.}-\frac{1}{4 !} F_{p\left[m_{1} m_{2} m_{3}\right.}\right) \partial_{m_{4}} \xi_{\left.m_{5} m_{6}\right]}
\end{aligned}
$$

and

$$
\delta \Xi_{p \mid m n q}=0, \quad \delta \Xi_{p \mid m_{1} \cdots m_{6}}=6 ! D_{p} D_{\left[m_{1}\right.} \xi_{\left.m_{2} \cdots m_{6}\right]} .
$$

As expected, these transformations contain second derivatives of the transformation parameters, in complete analogy with the transformation of the usual affine connection under ordinary diffeomorphisms.

\section{Generalised diffeomorphisms}

The $\mathrm{E}_{7(7)}$ generalised Lie derivative [8] incorporates the usual seven-dimensional spatial diffeomorphisms as well as the gauge transformations of the 3 - and 6-form fields. Indeed, it is immediately obvious from the explicit expressions (2.3)-(2.6) that the generalised vielbein is not invariant under such gauge transformations, and that the transformation properties can be read off directly from the components, equations (2.17) and (2.18). We will therefore combine all these transformations and internal diffeomorphisms into a generalised Lie derivative of the 56 -bein $\mathcal{V}$, such that

$$
\delta_{\Lambda} \mathcal{V}_{\mathcal{M} A B}=\hat{\mathcal{L}}_{\Lambda} \mathcal{V}_{\mathcal{M} A B}
$$

where $[8,9]^{6}$

$$
\hat{\mathcal{L}}_{\Lambda} X_{\mathcal{M}}=\frac{1}{2} \Lambda^{\mathcal{N}} \partial_{\mathcal{N}} X_{\mathcal{M}}+6\left(t^{\alpha}\right)_{\mathcal{M}}^{\mathcal{N}}\left(t_{\alpha}\right)_{\mathcal{P}}{ }^{\mathcal{Q}} \partial_{\mathcal{Q}} \Lambda^{\mathcal{P}} X_{\mathcal{N}}
$$

\footnotetext{
${ }^{6}$ The first prefactor is introduced for convenience: $\frac{1}{2} \Lambda^{\mathcal{M}} \partial_{\mathcal{M}} \equiv \Lambda^{m 8} \partial_{m 8}+\cdots \equiv \Lambda^{m} \partial_{m}+\cdots$.
} 
as is usually done in generalised geometry [8, 9, 31, 45, 46]. For a generalised covector density of weight $w$, this formula generalises to

$$
\hat{\mathcal{L}}_{\Lambda}^{(w)} X_{\mathcal{M}}=\frac{1}{2} \Lambda^{\mathcal{N}} \partial_{\mathcal{N}} X_{\mathcal{M}}+6\left(t^{\alpha}\right)_{\mathcal{M}}{ }^{\mathcal{N}}\left(t_{\alpha}\right)_{\mathcal{P}}{ }^{\mathcal{Q}} \partial_{\mathcal{Q}} \Lambda^{\mathcal{P}} X_{\mathcal{N}}+\frac{1}{2} w \partial_{\mathcal{N}} \Lambda^{\mathcal{N}} X^{\mathcal{M}}
$$

Note that generalised tensor densities are tensors that transform non-trivially under the $\mathbb{R}^{+}$factor of $\mathrm{E}_{d(d)} \times \mathbb{R}^{+}$in the language of [8]. Thus the generalised vielbein $\mathcal{V}$ has weight zero. These relations are very suggestive of 56 internal coordinates $y^{\mathcal{M}}$, rather than only the seven internal coordinates $y^{m}$ coming from $D=11$ supergravity. However, it should be understood that all relations are valid only in conjunction with the section condition ${ }^{7}[8,9]$

$$
t_{\alpha}^{\mathcal{M N}} \partial_{\mathcal{M}} \otimes \partial_{\mathcal{N}}=0, \quad \Omega^{\mathcal{M N}} \partial_{\mathcal{M}} \otimes \partial_{\mathcal{N}}=0 .
$$

This condition is crucial in order for the algebra of generalised gauge transformations to be properly defined. In fact, the closure of the algebra and the Jacobi identity only hold if the above condition is satisfied [8, 9, 46, 48]. This condition also allows one to introduce the extra structure associated with extra coordinates, and in an $\mathrm{E}_{7(7)}$ covariant manner, without having to view eleven-dimensional supergravity as a bona fide KaluzaKlein reduction of yet another genuinely higher dimensional theory (which does not appear to exist). Therefore, the requirement that

$$
\partial_{\mathcal{M}}= \begin{cases}\partial_{m} & \text { if } \mathcal{M}=m 8 \\ 0 & \text { otherwise }\end{cases}
$$

is not a reduction ansatz, but simply a solution of constraint (4.4). Another solution of the section condition leads to type IIB theory $[8,10,17,49,50]$. In this way by extending the coordinates one can unify these various descriptions in a single framework. In this work, we will always assume equation (4.5), reducing to a generalised geometric framework in the sense of Hitchin and Gualtieri [51, 52].

In order to see the link with the explicit formulae at the end of section 2.1, we now decompose the gauge transformation parameter in terms of the GL(7) subgroup as follows:

$$
\Lambda^{\mathcal{M}}=\left(\Lambda^{m}, \Lambda_{m n}, \Lambda^{p q}, \Lambda_{p}\right)
$$

Clearly, we can identify $\Lambda^{m}$ as the diffeomorphism parameter and $\Lambda_{m n}$ as the gauge parameter of three-form gauge transformations. Dualising $\Lambda^{p q}$ to a 5 -form allows us to identify this as the gauge parameter for 6 -form transformations. The final component $\Lambda_{p} \equiv \Lambda_{p 8}=-\Lambda_{8 p}$ is less understood. However, it is clearly related to gauge transformations associated with dual gravity. Although, we do not have a good understanding (at least not beyond the linearised level) of what these gauge transformations could involve, this does not cause us any problems. This is because dual gravity degrees of freedom do not contribute to the 56-bein. Equivalently, the 70 scalars in the four-dimensional theory have no contribution from the dualisation of gravitational degrees of freedom. Therefore, we would expect that

\footnotetext{
${ }^{7}$ The section condition first appeared in the context of $\mathrm{O}(d, d)[47]$, where it is related to the levelmatching condition in bosonic string theory.
} 
the generalised gauge transformation of the 56-bein with respect to $\Lambda_{p}$ transformations vanishes. This can be shown simply: assume that

$$
\Lambda^{\mathcal{M}}=\left(0,0,0, \Lambda_{p}\right) .
$$

Using equation (4.2), the generalised Lie derivative of the 56-bein reduces to

$$
\hat{\mathcal{L}}_{\Lambda} \mathcal{V}_{\mathcal{M} A B}=24\left(t^{\alpha}\right)_{\mathcal{M}}^{\mathcal{N}}\left(t_{\alpha}\right)^{p 8 q 8} \partial_{q} \Lambda_{p} \mathcal{V}_{\mathcal{N} A B}
$$

But, we know that

$$
\left(t_{\alpha}\right)^{p 8 q 8}=\left(t_{\alpha}\right)^{[p 8 q 8]}=0 .
$$

Hence, the 56-bein does not transform with respect to $\Lambda_{p}$ gauge transformations.

Similarly, it is straightforward to check that (4.1) precisely reproduces the coordinate and gauge transformations, (2.14), (2.17) and (2.18) with

$$
\Lambda^{m}=\xi^{m}, \quad \Lambda_{m n}=12 \sqrt{2} \xi_{m n}, \quad \Lambda^{m n}=6 ! \sqrt{2} \eta^{m n p_{1} \cdots p_{5}} \xi_{p_{1} \cdots p_{5}},
$$

where $\xi_{m n}$ and $\xi_{m_{1} \ldots m_{5}}$ are the 2 -form and 5 -form gauge parameters, see equations (2.15) and (2.16). As an example, consider the transformation of component $\mathcal{V}_{m n} A B$ :

$$
\begin{aligned}
\delta_{\Lambda} \mathcal{V}_{m n A B} & =\hat{\mathcal{L}}_{\Lambda} \mathcal{V}_{m n A B}=\frac{1}{2} \Lambda^{\mathcal{N}} \partial_{\mathcal{N}} \mathcal{V}_{m n A B}+6\left(t^{\alpha}\right)_{m n}{ }^{\mathcal{N}}\left(t_{\alpha}\right)_{\mathcal{P}}{ }^{\mathcal{Q}_{2}} \partial_{\mathcal{Q}} \Lambda^{\mathcal{P}} \mathcal{V}_{\mathcal{N} A B} \\
& =\Lambda^{p} \partial_{p} \mathcal{V}_{m n A B}+12\left(t^{\alpha}\right)_{m n}{ }^{\mathrm{RS}}\left(t_{\alpha}\right)_{\mathrm{PQ}}{ }^{q 8} \partial_{q} \Lambda^{\mathrm{PQ}} \mathcal{V}_{\mathrm{RS} A B}+12\left(t^{\alpha}\right)_{m n R \mathrm{RS}}\left(t_{\alpha}\right)^{\mathrm{PQ} q 8} \partial_{q} \Lambda_{\mathrm{PQ}} \mathcal{V}^{\mathrm{RS}}{ }_{A B} \\
& =\Lambda^{p} \partial_{p} \mathcal{V}_{m n A B}-4\left(\delta_{\mathrm{Q}[n}^{\mathrm{RS}} \delta_{m] \mathrm{P}}^{q 8}-1 / 8 \delta_{m n}^{\mathrm{RS}} \delta_{\mathrm{PQ}}^{q 8}\right) \partial_{q} \Lambda^{\mathrm{PQ}} \mathcal{V}_{\mathrm{RS} A B}+6 \delta_{m n \mathrm{RS}}^{\mathrm{PQ} q 8} \partial_{q} \Lambda_{\mathrm{PQ}} \mathcal{V}_{A B}^{\mathrm{RS}} \\
& =\left(\xi^{p} \partial_{p} \mathcal{V}_{m n A B}+2 \partial_{[m} \xi^{q} \mathcal{V}_{q] n A B}-\frac{1}{2} \partial_{p} \xi^{p} \mathcal{V}_{m n A B}\right)+36 \sqrt{2} \partial_{[p} \xi_{m n]} \mathcal{V}_{A B}^{p},
\end{aligned}
$$

where we have made use of the $\mathrm{E}_{7(7)}$ representation given in appendix $\mathrm{A}$. As can be verified by a direct computation using the definition of $\mathcal{V}_{m n A B}$ given in (2.4), this corresponds to the transformation of $\mathcal{V}_{m n} A B$ under coordinate and 3-form gauge transformations, (2.17). Verifying the precise agreement between (4.1) and the formulae derived in section 2.1 for the remaining components is equally straightforward.

We require that the generalised Lie derivative $\hat{\mathcal{L}}$ satisfies the product rule and that a generalised scalar transforms as

$$
\hat{\mathcal{L}}_{\Lambda} S=\frac{1}{2} \Lambda^{\mathcal{N}} \partial_{\mathcal{N}} S
$$

from which the Lie derivative of any generalised tensor can be found. In particular,

$$
\hat{\mathcal{L}}_{\Lambda} X^{\mathcal{M}}=\frac{1}{2} \Lambda^{\mathcal{N}} \partial_{\mathcal{N}} X^{\mathcal{M}}-6\left(t^{\alpha}\right)_{\mathcal{N}}^{\mathcal{M}}\left(t_{\alpha}\right)_{\mathcal{P}}{ }^{\mathcal{Q}} \partial_{\mathcal{Q}} \Lambda^{\mathcal{P}} X^{\mathcal{N}}
$$

This encodes the gauge transformations of the components of vector fields $\mathcal{B}_{\mu}^{\mathcal{M}}$, equations (2.25) and (2.26). However, in order to obtain the correct coordinate transformations, (2.24) and (2.27), one must identify $\mathcal{B}_{\mu}^{\mathcal{M}}$ as a generalised tensor density of weight $1 / 2$, i.e.

$$
\hat{\mathcal{L}}_{\Lambda} \mathcal{B}_{\mu}^{\mathcal{M}}=\frac{1}{2} \Lambda^{\mathcal{N}} \partial_{\mathcal{N}} \mathcal{B}_{\mu}^{\mathcal{M}}-6\left(t^{\alpha}\right)_{\mathcal{N}}^{\mathcal{M}}\left(t_{\alpha}\right)_{\mathcal{P}}{ }^{\mathcal{Q}} \partial_{\mathcal{Q}} \Lambda^{\mathcal{P}} \mathcal{B}_{\mu}^{\mathcal{N}}+\frac{1}{4} \partial_{\mathcal{N}} \Lambda^{\mathcal{N}} \mathcal{B}_{\mu}^{\mathcal{M}}
$$


Furthermore, we can use equation (4.12) to find the transformation of $\mathcal{B}_{\mu m}$ under 2form and 5 -form gauge transformations, equation (2.28). Thus, we can deduce that the transformation of $A_{\mu n_{1} \ldots n_{7}, m}$ under 2-form and 5 -form gauge transformations is given by equations (2.29) and (2.30), respectively.

\section{Generalised vielbein postulates and generalised geometry}

The external GVPs, equations (3.1)-(3.4), can be identified as the components of a single equation satisfied by $\mathcal{V},{ }^{8}$

$$
\partial_{\mu} \mathcal{V}_{\mathcal{M} A B}+2 \hat{\mathcal{L}}_{\mathcal{B}_{\mu}} \mathcal{V}_{\mathcal{M} A B}+\mathcal{Q}_{\mu}^{C}\left[A \mathcal{V}_{\mathcal{M} B] C}=\mathcal{P}_{\mu A B C D} \mathcal{V}_{\mathcal{M}}{ }^{C D}\right.
$$

where $\hat{\mathcal{L}}$ is the $\mathrm{E}_{7(7)}$ generalised Lie derivative defined in equation (4.2), or more specifically,

$$
\hat{\mathcal{L}}_{\mathcal{B}_{\mu}} \mathcal{V}_{\mathcal{M} A B}=\frac{1}{2} \mathcal{B}_{\mu}{ }^{\mathcal{N}} \partial_{\mathcal{N}} \mathcal{V}_{\mathcal{M} A B}+6\left(t^{\alpha}\right)_{\mathcal{M}}{ }^{\mathcal{N}}\left(t_{\alpha}\right)_{\mathcal{P}}{ }^{\mathcal{Q}} \partial_{\mathcal{Q}} \mathcal{B}_{\mu}{ }^{\mathcal{P}} \mathcal{V}_{\mathcal{N} A B}
$$

We note that the combination

$$
\partial_{\mu}+2 \hat{\mathcal{L}}_{\mathcal{B}_{\mu}}
$$

already appears in reference [10] (see (2.27) of ref. [10]), where it is introduced as the covariant derivative with respect to $x$-dependent generalised diffeomorphisms. It is now straightforward to check that (5.1) indeed coincides component by component with equations (3.1)-(3.4).

In a four-dimensional maximal gauged theory the scalars satisfy a Cartan equation of the form $[22]$

$$
\partial_{\mu} \mathcal{V}_{\mathcal{M} i j}-g \mathcal{B}_{\mu}{ }^{\mathcal{P}} X_{\mathcal{P} \mathcal{M}}{ }^{\mathcal{N}} \mathcal{V}_{\mathcal{N} i j}+\mathcal{Q}_{\mu[i}^{k} \mathcal{V}_{\mathcal{M} j] k} \mathcal{V}_{\mathcal{N} i j}=\mathcal{P}_{\mu i j k l} \mathcal{V}_{\mathcal{M}}{ }^{k l}
$$

where $X_{\mathcal{M}}$ generate the gauge algebra. Comparing the eleven-dimensional equation (5.1) with the four-dimensional equation (5.3) to which it reduces under reduction, we find that from an eleven-dimensional point of view, the generators of the gauge algebra are given by ${ }^{9}$

$$
\mathcal{B}_{\mu}^{\mathcal{P}} X_{\mathcal{P} \mathcal{M}}{ }^{\mathcal{N}} \mathcal{V}_{\mathcal{N} i j}=-2 \hat{\mathcal{L}}_{\mathcal{B}_{\mu}} \mathcal{V}_{\mathcal{M} A B}
$$

Hence, the higher-dimensional origin of the embedding tensor is read off from the external GVPs [1] that directly reduce to the Cartan equation of the maximally gauged four-dimensional theory that defines the embedding tensor as the gauge generators as anticipated in [1] and explicitly verified for the $S^{7}$ and Scherk-Schwarz reductions in $[6,7]$. In refs. $[8,27,53,54]$ the embedding tensor is argued to be related to the generalised Lie derivative by group theory arguments and consideration of the scalar potential.

\footnotetext{
${ }^{8}$ Recall that throughout this paper, we assume a solution of the $E_{7(7)}$ section condition of the form

$$
\partial_{\mathcal{M}} \neq 0 \quad \text { for } \mathcal{M}=m, \quad \text { otherwise } 0 .
$$
}

${ }^{9}$ We would like to thank Henning Samtleben for discussions on this. 
The internal GVPs, (3.9)-(3.15), likewise can be viewed as defining generalised connections: they can be written compactly as

$$
\partial_{m} \mathcal{V}_{\mathcal{M} A B}-\boldsymbol{\Gamma}_{m \mathcal{M}} \mathcal{N}^{\mathcal{V} A B}+\mathcal{Q}_{m[A}^{C} \mathcal{V}_{\mathcal{M} B] C}=\mathcal{P}_{m A B C D} \mathcal{V}_{\mathcal{M}}{ }^{C D}
$$

In this case, the generalised affine connection $\Gamma_{\mathcal{M N}}{ }^{\mathcal{P}}$ is non-zero only for the components with $\mathcal{M}=m$, which is the component appearing in equation (5.5). With this restriction, it can be decomposed as

$$
\boldsymbol{\Gamma}_{m \mathcal{M}^{\mathcal{N}}}=\boldsymbol{\Gamma}_{m}^{\alpha}\left(t_{\alpha}\right)_{\mathcal{M}^{\mathcal{N}}}
$$

It thus takes values in the Lie algebra of $\mathrm{E}_{7(7)}$, in analogy with the usual affine connection that takes values in the Lie algebra of $\operatorname{GL}(n)$; hence we can write

$$
\boldsymbol{\Gamma}_{m}{ }^{\alpha} \equiv\left\{\left(\boldsymbol{\Gamma}_{m}\right)_{\mathrm{M}}^{\mathrm{N}},\left(\boldsymbol{\Gamma}_{m}\right)^{\mathrm{MNPQ}}\right\}
$$

In particular, we also define

$$
\left(\boldsymbol{\Gamma}_{m}\right)_{\mathrm{MNPQ}} \equiv \frac{1}{24} \epsilon_{\mathrm{MNPQRSTU}}\left(\boldsymbol{\Gamma}_{m}\right)^{\mathrm{RSTU}} .
$$

The components of the generalised affine connection $\boldsymbol{\Gamma}_{m} \mathcal{N}^{\mathcal{P}}$ can be read off by direct comparison with equations (3.9)-(3.15); the non-zero components are

$$
\begin{aligned}
& \left(\boldsymbol{\Gamma}_{m}\right)_{p 8}^{q 8}=-\left(\boldsymbol{\Gamma}_{m}\right)^{q 8}{ }_{p 8}=\frac{1}{2} \Gamma_{m p}^{q}+\frac{1}{4} \Gamma_{m n}^{n} \delta_{p}^{q}, \\
& \left(\boldsymbol{\Gamma}_{m}\right)_{p q}{ }^{r s}=-\left(\boldsymbol{\Gamma}_{m}\right)^{r s}{ }_{p q}=2 \Gamma_{m[p}^{[r} \delta_{q]}^{s]}-\frac{1}{2} \Gamma_{m n}^{n} \delta_{p q}^{r s}, \\
& \left(\boldsymbol{\Gamma}_{m}\right)_{p 8}^{r s}=-\left(\boldsymbol{\Gamma}_{m}\right)^{r s}=3 \sqrt{2} \eta^{r s t_{1} \cdots t_{5}} \Xi_{m \mid p t_{1} \cdots t_{5}}, \\
& \left(\boldsymbol{\Gamma}_{m}\right)_{p q r 8}=\left(\boldsymbol{\Gamma}_{m}\right)_{r 8 p q}=3 \sqrt{2} \Xi_{m \mid p q r}, \\
& \left(\boldsymbol{\Gamma}_{m}\right)^{p q r s}=\frac{1}{\sqrt{2}} \eta^{p q r s t_{1} t_{2} t_{3}} \Xi_{m \mid t_{1} t_{2} t_{3}},
\end{aligned}
$$

where $\Gamma_{m n}^{p}$ is the usual affine connection for the seven internal directions. Equivalently, the non-vanishing components of $\boldsymbol{\Gamma}_{\mathcal{M}}{ }^{\alpha}$ are

$$
\begin{aligned}
\left(\boldsymbol{\Gamma}_{m}\right)_{n}{ }^{p} & \equiv-\Gamma_{m n}^{p}+\frac{1}{4} \delta_{n}^{p} \Gamma_{m q}^{q}, & \left(\boldsymbol{\Gamma}_{m}\right)_{8}^{8} & =-\frac{3}{4} \Gamma_{m n}^{n}, \\
\left(\boldsymbol{\Gamma}_{m}\right)_{8}{ }^{n} & =\sqrt{2} \eta^{n p_{1} \cdots p_{6}} \Xi_{m \mid p_{1} \cdots p_{6}}, & \left(\boldsymbol{\Gamma}_{m}\right)^{n_{1} \cdots n_{4}} & =\frac{1}{\sqrt{2}} \eta^{n_{1} \cdots n_{4} p_{1} p_{2} p_{3}} \Xi_{m \mid p_{1} p_{2} p_{3}} .
\end{aligned}
$$

Note that as required by the SL(8) property of the indices, we have $\left(\boldsymbol{\Gamma}_{m}\right)_{\mathrm{M}}{ }^{\mathrm{M}}=0$.

From a generalised geometry viewpoint, there is in principle no reason why a generalised connection $\Gamma_{\mathcal{M N}}{ }^{\mathcal{P}}$ cannot be non-zero for other values of $\mathcal{M}$ (as in ref. [8]) such that

$$
\partial_{\mathcal{M}} \mathcal{V}_{\mathcal{N} A B}-\Gamma_{\mathcal{M N}}{ }^{\mathcal{P}} \mathcal{V}_{\mathcal{P} A B}+\mathcal{Q}_{\mathcal{M}[A}^{C} \mathcal{V}_{\mathcal{N} B] C}=\mathcal{P}_{\mathcal{M} A B C D} \mathcal{V}_{\mathcal{N}}{ }^{C D}
$$

In our approach, the generalised connection is given to us by the equations that come from $D=11$ supergravity, or more precisely the on-shell equivalent $\mathrm{SU}(8)$ reformulation 
thereof. However, as in usual differential geometry there is a freedom in redefining the connections. For example, one such freedom is the fact that the affine and spin connections can be redefined using a covariant tensor in such a way that the vielbein postulate remains unchanged. Analogously, such a redefinition with a generalised tensor with nonzero components in all 56 directions can be used to "excite" the other components of the generalised connections. Ultimately different redefinitions, while physically equivalent, will be motivated by different contexts. For example, there are redefinitions of the connection in which the scalar potential is purely given in terms of a generalised curvature scalar rather than the generalised curvature scalar and the square of the non-metricity $P_{m}$ (see section 6). Moreover, there is an additional freedom in redefining the generalised connections, which from an eleven-dimensional perspective corresponds to a redefinition that leaves the fermion supersymmetry transformations unchanged [26]. In any case, $D=11$ supergravity, as manifested in the $\mathrm{SU}(8)$ reformulation, leads us to conclude that, up to redefinitions, any covariant derivative that acts on the generalised vielbein only has components along the usual seven-dimensional space. We also note that $\mathrm{E}_{7(7)}$ valuedness of the affine connection implies

$$
\mathcal{D}_{\mathcal{M}} \Omega_{\mathcal{N P}}=0
$$

The transformation of the generalised affine connection under generalised diffeomorphisms is

$$
\delta_{\Lambda} \boldsymbol{\Gamma}_{\mathcal{M N}}{ }^{\mathcal{P}}=\left(\hat{\mathcal{L}}_{\Lambda}^{(-1 / 2)} \boldsymbol{\Gamma}\right)_{\mathcal{M N}}{ }^{\mathcal{P}}+6\left(t^{\alpha}\right)_{\mathcal{N}}^{\mathcal{P}}\left(t_{\alpha}\right)_{\mathcal{Q}}^{\mathcal{R}} \partial_{\mathcal{M}} \partial_{\mathcal{R}} \Lambda^{\mathcal{Q}}
$$

where $\hat{\mathcal{L}}_{\Lambda}^{(-1 / 2)} \boldsymbol{\Gamma}$ is the canonical generalised Lie derivative of $\boldsymbol{\Gamma}$ with weight $-1 / 2$ along $\Lambda$, equation (4.3). The above transformation encodes the usual inhomogeneous transformation of the affine connection as well as the gauge transformations of $\Xi$, which include second derivatives of the 2-form and 5-form gauge parameters, equations (3.24) and (3.25).

When viewed as an analogue of the vielbein postulate, the internal GVP, (5.5), furnishes an $\mathrm{E}_{7(7)}$ and $\mathrm{SU}(8)$ covariant derivative along $y^{m}$. The generalised affine connection transforms in exactly such a way, (5.13), so that given a generalised vector density $X^{\mathcal{M}}$ of weight $w$,

$$
\mathcal{D}_{\mathcal{M}} X^{\mathcal{N}} \equiv \partial_{\mathcal{M}} X^{\mathcal{N}}+\boldsymbol{\Gamma}_{\mathcal{M P}}{ }^{\mathcal{N}} X^{\mathcal{P}}-\frac{2}{3} w \boldsymbol{\Gamma}_{\mathcal{P} \mathcal{M}}{ }^{\mathcal{P}} X^{\mathcal{N}}
$$

transforms as a generalised tensor density of weight $(w-1 / 2)$ (note the order of indices in the last term, which is $\boldsymbol{\Gamma}_{\mathcal{P} \mathcal{M}}{ }^{\mathcal{P}}$, and not $\boldsymbol{\Gamma}_{\mathcal{M P}}{ }^{\mathcal{P}}$ ). Observe that the weight term in the covariant derivative of a generalised tensor density differs from the usual covariant derivative of a tensor density because of the way $\boldsymbol{\Gamma}_{\mathcal{P} \mathcal{M}}{ }^{\mathcal{P}}$ transforms under generalised diffeomorphisms, equation (5.13). The fact that the covariant derivative of a tensor density must itself be a tensor density with weight $1 / 2$ less than the weight of the original tensor must be true of any covariant derivative that is defined in $\mathrm{E}_{7(7)}$ generalised geometry. To see why this is true consider the non-covariant terms in the transformation of $\partial_{\mathcal{M}} X^{\mathcal{N}}$, where $X^{\mathcal{M}}$ is a generalised tensor:

$$
\frac{1}{2} \partial_{\mathcal{M}} \Lambda^{\mathcal{Q}} \partial_{\mathcal{Q}} X^{\mathcal{N}}-6\left(t^{\alpha}\right)_{\mathcal{M}}{ }^{\mathcal{Q}}\left(t_{\alpha}\right)_{\mathcal{P}}{ }^{\mathcal{R}} \partial_{\mathcal{R}} \Lambda^{\mathcal{P}} \partial_{\mathcal{Q}} X^{\mathcal{N}}-6\left(t^{\alpha}\right)_{\mathcal{Q}^{\mathcal{N}}}\left(t_{\alpha}\right)_{\mathcal{P}}{ }^{\mathcal{R}} \partial_{\mathcal{M}} \partial_{\mathcal{R}} \Lambda^{\mathcal{P}} X^{\mathcal{Q}}
$$


The third term in the expression above must be cancelled by an inhomogeneous term in the transformation of the connection. However, using [10]

$$
\left(t^{\alpha}\right)_{\mathcal{M}}^{\mathcal{N}}\left(t_{\alpha}\right)_{\mathcal{P}} \mathcal{Q}=\frac{1}{12} \delta_{\mathcal{M}}^{\mathcal{Q}} \delta_{\mathcal{P}}^{\mathcal{N}}+\frac{1}{24} \delta_{\mathcal{M}}^{\mathcal{N}} \delta_{\mathcal{P}}^{\mathcal{Q}}+\left(t^{\alpha}\right)_{\mathcal{M P}}\left(t_{\alpha}\right)^{\mathcal{N} \mathcal{Q}}-\frac{1}{24} \Omega_{\mathcal{M P}} \Omega^{\mathcal{N} \mathcal{Q}}
$$

and the section condition (4.4), the remaining terms give

$$
-\frac{1}{4} \partial_{\mathcal{Q}} \Lambda^{\mathcal{Q}} \partial_{\mathcal{M}} X^{\mathcal{N}}
$$

hence the covariant derivative of $X^{\mathcal{M}}, \mathcal{D}_{\mathcal{M}} X^{\mathcal{N}}$, must have weight $-1 / 2$ less than $X^{\mathcal{M}}$ itself. ${ }^{10}$ This is also the case in $\mathrm{E}_{d(d)} \times \mathbb{R}^{+}$generalised geometry [8] where the connection naturally lowers the weight of the tensor because generalised vectors are weighted. ${ }^{11}$

\section{$6 \quad$ Generalised $\mathrm{E}_{7(7)}$ curvature}

The generalised covariant derivative defined above can be used to define a generalised curvature (generalised Riemann tensor) $\mathbf{R}$, given by

$$
\left[\mathcal{D}_{\mathcal{M}}, \mathcal{D}_{\mathcal{N}}\right] X_{\mathcal{P}}=\mathbf{R}_{\mathcal{M N} \mathcal{P}}{ }^{\mathcal{Q}} X_{\mathcal{Q}}
$$

Note that because of the transformation property of the covariant derivative, the second covariant derivative acts on a generalised tensor density of weight $-1 / 2$ (assuming that $X^{\mathcal{P}}$ is a generalised tensor, and thus of weight zero). Hence the generalised Riemann tensor is in fact a tensor density of weight -1 . Furthermore, using equation (5.14)

$$
3 \boldsymbol{\Gamma}_{[\mathcal{M N}]^{\mathcal{Q}}} \partial_{\mathcal{Q}} X_{\mathcal{P}}=\boldsymbol{\Gamma}_{\mathcal{Q}[\mathcal{M}}{ }^{\mathcal{Q}} \partial_{\mathcal{N}]} X_{\mathcal{P}}
$$

(which follows directly from the explicit expressions for the components in (5.9)), the fact that the covariant derivative modifies the weight of the generalised tensor is crucial in cancelling

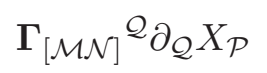

from the commutator of the covariant derivatives in equation (6.1). It is important that the term above is cancelled because from equation (5.13) the antisymmetrisation (in $\mathcal{M}$ and $\mathcal{N}$ ) of the generalised connection is not covariant - this is unlike ordinary differential geometry where the antisymmetrisation of an affine connection can be identified as a covariant torsion. In fact, a generalised torsion $\mathbf{T}_{\mathcal{M N}}{ }^{\mathcal{P}}$, as defined by

$$
\left[\mathcal{D}_{\mathcal{M}}, \mathcal{D}_{\mathcal{N}}\right] S=\mathbf{T}_{\mathcal{M N}}{ }^{\mathcal{P}} \partial_{\mathcal{P}} S
$$

for some scalar $S$, can simply be shown to vanish in our scheme. Hence, the absence of a torsion term on the right hand side of equation (6.1). Let us emphasize once again that it is the explicit knowledge of the affine connection coefficients in (5.9) that enables us to overcome and resolve this well known difficulty encountered in previous work, see e.g. [55].

\footnotetext{
${ }^{10}$ The argument is essentially the same if $X^{\mathcal{M}}$ is a generalised tensor density.

${ }^{11}$ We would like to thank Dan Waldram for correspondence on this point.
} 
Computing the left hand side of equation (6.1) using the definition of the covariant derivative (5.14), gives the form of the Riemann tensor, which turns out to be analogous to the expression for the conventional Riemann tensor in terms of an affine torsion-free connection

$$
\mathbf{R}_{\mathcal{M N \mathcal { P }}}^{\mathcal{Q}}=-2 \partial_{[\mathcal{M}} \boldsymbol{\Gamma}_{\mathcal{N}] \mathcal{P}}{ }^{\mathcal{Q}}+2 \boldsymbol{\Gamma}_{[\mathcal{M} \mid \mathcal{P}}{ }^{\mathcal{R}} \boldsymbol{\Gamma}_{\mid \mathcal{N}] \mathcal{R}}{ }^{\mathcal{Q}}
$$

Since the generalised Riemann tensor is defined using covariant derivatives, it is by definition an object that transforms covariantly under generalised diffeomorphisms, up to weight terms. However, in appendix B, we explicitly verify that it transforms covariantly under generalised diffeomorphisms as a generalised tensor density of weight -1 .

The non-zero components of the generalised Riemann curvature $\mathbf{R}_{\mathcal{M N \mathcal { P }}}{ }^{\mathcal{Q}}$ can be directly computed from equations (5.9); they are

$$
\begin{aligned}
& \mathbf{R}_{m 8 n 8 p 8}{ }^{q 8}=-\mathbf{R}_{m 8 n 8}{ }^{q 8}{ }_{p 8}=\frac{1}{2} R_{m n p}{ }^{q}, \\
& \mathbf{R}_{m 8 n 8 p q}{ }^{r s}=-\mathbf{R}_{m 8 n 8^{r s}}{ }_{p q}=2 R_{m n[p}{ }^{[r} \delta_{q]}^{s]}, \\
& \mathbf{R}_{m 8 n 8 p 8}{ }^{r s}=-\mathbf{R}_{m 8 n 8}{ }^{r s}{ }_{p 8}=2 \sqrt{2} \delta_{p}^{[r} \eta^{s] t_{1} \cdots t_{6}} D_{[m} \Xi_{n] \mid t_{1} \cdots t_{6}} \\
& +6 \eta^{r s t_{1} \cdots t_{5}} \Xi_{\left[m|| t_{1} t_{2} p\right.} \Xi_{\mid n] \mid t_{4} t_{5} t_{6}}, \\
& \mathbf{R}_{m 8 n 8 p q r 8}=\mathbf{R}_{m 8 n 8 r 8 p q}=-6 \sqrt{2} D_{[m} \Xi_{n] \mid p q r}, \\
& \mathbf{R}_{m 8 n 8}^{p q r s}=-\sqrt{2} \eta^{p q r s t_{1} t_{2} t_{3}} D_{[m} \Xi_{n] \mid t_{1} t_{2} t_{3}} .
\end{aligned}
$$

Equivalently, decomposing $\mathbf{R}$ as

$$
\mathbf{R}_{\mathcal{M N \mathcal { P }}}{ }^{\mathcal{Q}}=\mathbf{R}_{\mathcal{M N}}{ }^{\alpha}\left(t_{\alpha}\right)_{\mathcal{P}}{ }^{\mathcal{Q}}, \quad \mathbf{R}_{\mathcal{M N}}{ }^{\alpha} \equiv\left\{\left(\mathbf{R}_{\mathcal{M N}}\right)_{\mathrm{M}}{ }^{\mathrm{N}},\left(\mathbf{R}_{\mathcal{M N}}\right)^{\mathrm{MNPQ}}\right\}
$$

the non-zero components of $\mathbf{R}_{\mathcal{M N}}{ }^{\alpha}$ are

$$
\begin{aligned}
\left(\mathbf{R}_{m 8 n 8}\right)_{p}^{q} & =-R_{m n p}{ }^{q}, \\
\left(\mathbf{R}_{m 8 n 8}\right)_{8}^{p} & =-2 \eta^{p t_{1} \cdots t_{6}}\left(\sqrt{2} D_{[m} \Xi_{n] \mid t_{1} \cdots t_{6}}-\Xi_{m \mid t_{1} t_{2} t_{3}} \Xi_{n \mid t_{4} t_{5} t_{6}}\right), \\
\left(\mathbf{R}_{m 8 n 8}\right)^{p_{1} \cdots p_{4}} & =-\sqrt{2} \eta^{p_{1} \cdots p_{4} r s t} D_{[m} \Xi_{n] \mid r s t} .
\end{aligned}
$$

Note that the above components of the generalised Riemann tensor are not invariant under 2- and 5-form gauge transformations. Indeed, this is to be expected since the definition of the generalised Riemann tensor as a generalised tensor ensures its covariance, rather than invariance, under generalised gauge transformations. While this may be antithetical to our usual notions of gauge transformations and how physical fields must accordingly transform, in a generalised geometric setting the appearance of gauge non-invariant terms should not come as a surprise, and one ought to view gauge transformations as being similar to coordinate transformations for which the notion of covariance, as well as invariance, exists. In fact, we have already encountered this novelty before in the definitions of the generalised vielbein $\mathcal{V}_{\mathcal{M} A B}$ and vectors $\mathcal{B}_{\mu}{ }^{\mathcal{M}}$. However, "gauge covariance" limits the dependence of gauge non-invariant terms in a generalised tensor to bare 3-form and 6-form potentials, 
or their gauge invariant field strengths. Therefore, the gauge potentials can only enter gauge non-invariant terms without any derivatives, as in the $\mathrm{E}_{7(7)} 56$-bein, and the fact that they do (and thus all non-covariant terms cancel in the expressions below) constitutes a non-trivial consistency check of our scheme. This claim can be explicitly verified for the generalised Riemann tensor by expressing the above components directly in terms of the 3 -form and 6 -form gauge potentials and their associated field strengths, using (3.21) and (3.22),

$$
\begin{aligned}
& \left(\mathbf{R}_{m 8 n 8}\right)_{8}{ }^{p}=-\eta^{p q_{1} \cdots q_{6}}\left(6 \sqrt{2} R_{m n q_{1}}{ }^{r} A_{r q_{2} \cdots q_{6}}-\frac{2 \sqrt{2}}{7 !} D_{[m} F_{n] q_{1} \cdots q_{6}}+\frac{1}{6} D_{[m} F_{n] q_{1} q_{2} q_{3}} A_{q_{4} q_{5} q_{6}}\right. \\
& \left.-3 R_{m n q_{1}}{ }^{r} A_{r q_{2} q_{3}} A_{q_{4} q_{5} q_{6}}-\frac{2}{(4 !)^{2}} F_{m q_{1} q_{2} q_{3}} F_{n q_{4} q_{5} q_{6}}\right), \\
& \left(\mathbf{R}_{m 8 n 8}\right)^{p_{1} \cdots p_{4}}=-\frac{3 \sqrt{2}}{2} \eta^{p_{1} \cdots p_{4} r s t}\left(R_{m n r}^{u} A_{u s t}-\frac{1}{36} D_{[m} F_{n] r s t}\right) .
\end{aligned}
$$

It can now be explicitly verified that the transformation of components of the generalised Riemann tensor under coordinate and gauge transformations precisely matches the transformation given by generalised diffeomorphisms, as expected. For example, consider the transformation of the following component of $\mathbf{R}_{\mathcal{M N P}}{ }^{\mathcal{Q}}$ :

$$
\mathbf{R}_{m 8 n 8^{p q r s}}
$$

given in (6.6). We find that its transformation as derived from the generalised Lie derivative is

$$
\delta \mathbf{R}_{m 8 n 8^{p q r s}}=\mathcal{L}_{\Lambda}^{(+1)}\left(\mathbf{R}_{m 8 n 8^{p q r s}}\right)-\frac{3}{4} \eta^{p q r s t u_{1} u_{2}} R_{m n t}{ }^{u_{3}} \partial_{\left[u_{1}\right.} \Lambda_{\left.u_{2} u_{3}\right]} .
$$

Hence, while the generalised curvature tensor is a generalised density of weight -1 , the component above transforms as a tensor density of weight +1 under usual coordinate transformation and it also transforms non-trivially under a 2-form gauge transformation. Noting that this component is equal to the expression given in equation (6.9) and using equation (4.8), which gives the relation between $\Lambda_{m n}$ and the 2-form gauge transformation parameter $\xi_{m n}$, we find a precise match.

Now, consider the contraction of the generalised Riemann curvature:

$$
\mathbf{R}_{\mathcal{M N}}=\mathbf{R}_{\mathcal{M P N}}{ }^{\mathcal{P}}
$$

It is simple to see that the only non-zero component of $\mathbf{R}_{\mathcal{M N}}$ is

$$
\mathbf{R}_{m 8 n 8}=R_{m n}
$$

Hence the only gauge-invariant objects that can be formed from the generalised Riemann tensor are the Ricci tensor and the Ricci scalar. Therefore, at the 2-derivative level the internal Ricci scalar can be obtained from the generalised Riemann tensor and the flux terms correspond to the trace of the square of $\mathcal{P}_{m}$. Together these would correspond to the potential. In this sense the way the potential would be written here is different to 
the approach in [12] where the potential is written as a sigma-model in terms of the generalised metric

$$
M_{\mathcal{M N}}=\mathcal{V}_{\mathcal{M}}{ }^{A B} \mathcal{V}_{\mathcal{N} A B}+\mathcal{V}_{\mathcal{N}}^{A B} \mathcal{V}_{\mathcal{M} A B}
$$

and both the Ricci scalar and the flux terms arise from the same terms. The affine connection defined in equation (5.10) is not metric compatible with the non-metricity given by $\mathcal{P}_{m}$. Redefining a new connection that absorbs the non-metricity, similar to a redefinition that can be made in usual differential geometry (see ref. [56] for a useful discussion of torsion and non-metricity in differential geometry), will result in the scalar potential being given solely by the generalised Ricci scalar, as in ref. [8]. ${ }^{12}$ We defer a discussion of how the generalised geometry determines the potential in terms of the $\mathrm{E}_{7(7)}$ structures given here to a future work.

\section{Discussion}

The issue of defining generalised differential geometric structures, such as connections and curvatures, associated with exceptional duality groups and using them to construct the dynamics is clearly an important one and has also been considered in refs. [8, 32, 55, 57].

In this paper, we use the formalism developed in ref. [1] to derive $E_{7(7)}$ generalised geometric structures, including generalised connections and curvatures as well as making explicit the higher dimensional origin of the embedding tensor, for which the GVP plays a central role. We derive the $\mathrm{E}_{7(7)}$ connection which is used to construct the generalised curvature tensor, from the internal GVP and ultimately the $D=11$ theory. Importantly, and apart from the generalisation of the affine and spin connections, the internal GVP is not just of the form $D \mathcal{V}=0$, but has an extra contribution from $\mathcal{P}_{m}$ (this vanishes in the absence of the 4-form and 7-form field strengths, however, and then the GVP reduces to the standard one). This is a main difference with the ansatz made in refs. [8,55]. Another notable feature of the generalised covariant derivative defined here, similar to that defined in ref. [8] and as opposed to that defined in ref. [55], is that it changes the weight of the resulting generalised tensor, which is crucial, from our perspective, in allowing a generalised Riemann tensor to be defined.

In general, the approach taken in refs. $[8,32,55,57]$ is to try to generalise geometric structures to exceptional geometry using notions taken from usual differential geometry, such as metric compatibility of the connection, while incorporating the novelty of generalised geometry. For example, in ref. [8], the index on the generalised connection that is associated with the derivative has components along extended tangent space directions, unlike the connection defined here - although this can be done in our case as well (see comments after equation (5.13)). However, the difference in approach allows us to find a new contribution to what can be viewed as a vielbein compatibility of the connection, namely, $\mathcal{P}_{m}$ as well as a generalised Riemann tensor that transforms covariantly under full generalised diffeomorphisms, as well as local SU(8) transformations. The existence of a generalised Riemann tensor that is unambiguously defined in terms of the physical fields has

\footnotetext{
${ }^{12}$ We would like to thank Malcolm Perry for pointing this out to us.
} 
been studied extensively in the generalised geometry literature associated to exceptional as well as $\mathrm{O}(d, d)$ duality $[8,47,58-63]$. The approach taken in these papers has been to define the connection using certain $\mathrm{E}_{7(7)}$ covariant conditions such as metric-compatibility and torsion-freeness. While these connections, which are not unique and contain ambiguous pieces, do not define non-ambiguous Riemann tensors (though a Riemann tensor can be defined), they can be used to uniquely define a generalised Ricci tensor and scalar that corresponds to the potential. The approach taken in this paper is to use the connections that are given by the $\mathrm{SU}(8)$ invariant reformulation of the $D=11$ theory, without any prejudice regarding any conditions that they ought to satisfy, to define the differential geometrical quantities.

While, at the two-derivative level, the only scalar constructible from the generalised Riemann tensor reduces to the usual internal Ricci scalar, at a higher-derivative level, other scalars can be constructed that have explicit dependence on the gauge potentials. These along with other scalars constructed from structures such as $\mathcal{P}_{m}$ may help in providing an understanding of higher-derivative corrections from a generalised geometric perspective. We will consider this possibility in the future.

The analysis performed in this paper can also be straightforwardly applied to the $3+8$ split of $D=11$ supergravity, pertinent to the $\mathrm{E}_{8(8)}$ duality group. Some preliminary results for this case have already been obtained in refs. [1,39], and we hope to extend these partial results in a future work.

\section{Acknowledgments}

We are grateful to Henning Samtleben and Olaf Hohm for discussions and explanations of their recent work, especially [10]. We are also grateful to Martin Cederwall, Axel Kleinschmidt, Malcolm Perry and Dan Waldram for discussions. We would like to thank Henning Samtleben and ENS Lyon for hospitality. H.G. and M.G. would like to thank the Max-Planck-Institut für Gravitationsphysik (AEI) and in particular H.N. for hospitality. H.G. and M.G. are supported by King's College, Cambridge. The work of H.N. is supported in part by the Gay-Lussac-Humboldt Prize.

\section{A $\quad \mathrm{E}_{7(7)}$ algebra and identities}

In this appendix, we list useful equations with regard to the $\mathrm{SL}(8)$ decomposition of the $\mathrm{E}_{7(7)}$ algebra:

$$
\begin{aligned}
\left(t^{\mathrm{M}}{ }_{\mathrm{N}}\right)^{\mathrm{PQ}}{ }_{\mathrm{RS}} & =2\left(\delta_{\mathrm{N}[\mathrm{S}}^{\mathrm{PQ}} \delta_{\mathrm{R}]}^{\mathrm{M}}-\frac{1}{8} \delta_{\mathrm{N}}^{\mathrm{M}} \delta_{\mathrm{RS}}^{\mathrm{PQ}}\right), & \left(t^{\mathrm{M}}\right)_{\mathrm{RS}}{ }^{\mathrm{PQ}} & =-2\left(\delta_{\mathrm{N}[\mathrm{S}}^{\mathrm{PQ}} \delta_{\mathrm{R}]}^{\mathrm{M}}-\frac{1}{8} \delta_{\mathrm{N}}^{\mathrm{M}} \delta_{\mathrm{RS}}^{\mathrm{PQ}}\right), \\
\left(t_{\mathrm{PQRS}}\right)^{\mathrm{T}_{1} \ldots \mathrm{T}_{4}} & =\delta_{\mathrm{PQRS}}^{\mathrm{T}_{1} \ldots \mathrm{T}_{4}}, & \left(t_{\mathrm{PQRS}}\right)_{\mathrm{T}_{1} \ldots \mathrm{T}_{4}} & =\frac{1}{4 !} \eta_{\mathrm{PQRST}_{1} \ldots \mathrm{T}_{4}}, \\
\kappa^{\mathrm{M}}{ }_{\mathrm{N}},{ }_{\mathrm{Q}}^{\mathrm{P}} & =12\left(\delta_{\mathrm{Q}}^{\mathrm{M}} \delta_{\mathrm{N}}^{\mathrm{P}}-\frac{1}{8} \delta_{\mathrm{N}}^{\mathrm{M}} \delta_{\mathrm{Q}}^{\mathrm{P}}\right), & \kappa_{\mathrm{MNPQ}, \mathrm{RSTU}} & =\frac{2}{4 !} \eta_{\mathrm{MNPQRSTU}}, \\
\left(\kappa^{-1}\right)_{\mathrm{N}}{ }^{\mathrm{M}},{ }_{\mathrm{Q}}^{\mathrm{P}} & =\frac{1}{12}\left(\delta_{\mathrm{Q}}^{\mathrm{M}} \delta_{\mathrm{N}}^{\mathrm{P}}-\frac{1}{8} \delta_{\mathrm{N}}^{\mathrm{M}} \delta_{\mathrm{Q}}^{\mathrm{P}}\right), & \left(\kappa^{-1}\right)^{\mathrm{MNPQ}, \mathrm{RSTU}} & =\frac{1}{2 \cdot 4 !} \eta^{\mathrm{MNPQRSTU}} .
\end{aligned}
$$

where $\kappa$ is the Cartan Killing form on $\mathrm{E}_{7(7)}$. 


\section{B Generalised covariance of the curvature tensor}

The generalised curvature tensor, defined in (6.5), is

$$
\mathbf{R}_{\mathcal{M N \mathcal { P }}}{ }^{\mathcal{Q}}=-2 \partial_{[\mathcal{M}} \boldsymbol{\Gamma}_{\mathcal{N}] \mathcal{P}}{ }^{\mathcal{Q}}+2 \boldsymbol{\Gamma}_{[\mathcal{M} \mid \mathcal{P}}{ }^{\mathcal{R}} \boldsymbol{\Gamma}_{\mid \mathcal{N}] \mathcal{R}}{ }^{\mathcal{Q}}
$$

In this appendix we show that $\mathbf{R}_{\mathcal{M N \mathcal { N }}}{ }^{\mathcal{Q}}$ as defined above indeed transforms as a generalised tensor density of weight -1 under the transformation of the generalised connection, given in equation (5.13),

$$
\delta_{\Lambda} \boldsymbol{\Gamma}_{\mathcal{M N}} \mathcal{P}^{\mathcal{P}}=\left(\hat{\mathcal{L}}_{\Lambda}^{(-1 / 2)} \boldsymbol{\Gamma}\right)_{\mathcal{M N}}{ }^{\mathcal{P}}+6\left(t^{\alpha}\right)_{\mathcal{N}}^{\mathcal{P}}\left(t_{\alpha}\right)_{\mathcal{Q}}^{\mathcal{R}} \partial_{\mathcal{M}} \partial_{\mathcal{R}} \Lambda^{\mathcal{Q}}
$$

Under generalised diffeomorphisms, the transformation of the generalised Riemann tensor is

$$
\begin{aligned}
\delta_{\Lambda} \mathbf{R}_{\mathcal{M N \mathcal { P }}}^{\mathcal{Q}}= & -2 \partial_{[\mathcal{M}} \delta_{\Lambda} \boldsymbol{\Gamma}_{\mathcal{N}] \mathcal{P}}{ }^{\mathcal{Q}}+2\left(\delta_{\Lambda} \boldsymbol{\Gamma}_{[\mathcal{M} \mid \mathcal{P}}{ }^{\mathcal{R}}\right) \boldsymbol{\Gamma}_{\mid \mathcal{N}] \mathcal{R}}{ }^{\mathcal{Q}}+2 \boldsymbol{\Gamma}_{[\mathcal{M} \mid \mathcal{P}}{ }^{\mathcal{R}}\left(\delta_{\Lambda} \boldsymbol{\Gamma}_{\mid \mathcal{N}] \mathcal{R}}{ }^{\mathcal{Q}}\right) \\
= & -2\left(\hat{\mathcal{L}}_{\Lambda}^{(-1 / 2)} \partial \boldsymbol{\Gamma}\right)_{\mathcal{M N \mathcal { N }}}^{\mathcal{Q}}+12\left(t^{\alpha}\right)_{[\mathcal{M} \mid}{ }^{\mathcal{R}}\left(t_{\alpha}\right)_{\mathcal{S}}{ }^{\mathcal{T}} \partial_{\mathcal{T}} \Lambda^{\mathcal{S}} \partial_{\mathcal{R}} \boldsymbol{\Gamma}_{\mid \mathcal{N}] \mathcal{P}}^{\mathcal{Q}}-\partial_{[\mathcal{M} \mid} \Lambda^{\mathcal{R}} \partial_{\mathcal{R}} \Lambda_{\mid \mathcal{N}] \mathcal{P}} \mathcal{Q} \\
& +\frac{1}{2} \partial_{[\mathcal{M} \mid} \partial_{\mathcal{R}} \Lambda^{\mathcal{R}} \boldsymbol{\Gamma}_{\mid \mathcal{N}] \mathcal{P}}{ }^{\mathcal{Q}}+12\left(t^{\alpha}\right)_{[\mathcal{M} \mid}{ }^{\mathcal{R}}\left(t_{\alpha}\right)_{\mathcal{S}}{ }^{\mathcal{T}} \partial_{\mid \mathcal{N}]} \partial_{\mathcal{T}} \Lambda^{\mathcal{S}} \boldsymbol{\Gamma}_{\mathcal{R} \mathcal{P}}^{\mathcal{Q}}+2\left(\hat{\mathcal{L}}_{\Lambda}^{(-1)} \boldsymbol{\Gamma} \cdot \boldsymbol{\Gamma}\right)_{\mathcal{M N \mathcal { N }}} \mathcal{Q}
\end{aligned}
$$

where the generalised Lie derivatives $\hat{\mathcal{L}}_{\Lambda}^{(-1 / 2)}$ on $\partial \boldsymbol{\Gamma}$ and $\hat{\mathcal{L}}_{\Lambda}^{(-1)}$ on $\boldsymbol{\Gamma}$ are defined in analogy with (4.3). Further evaluation of this expression by means of the $\mathrm{E}_{7(7)}$ identity (5.16) and the section condition (4.4) yields

$$
\begin{aligned}
\delta_{\Lambda} \mathbf{R}_{\mathcal{M N \mathcal { P }}}{ }^{\mathcal{Q}} & =-2\left(\hat{\mathcal{L}}_{\Lambda}^{(-1 / 2)} \partial \boldsymbol{\Gamma}\right)_{\mathcal{M N \mathcal { P }}}{ }^{\mathcal{Q}}+\frac{1}{2} \partial_{\mathcal{R}} \Lambda^{\mathcal{R}} \partial_{[\mathcal{M} \mid} \boldsymbol{\Gamma}_{\mid \mathcal{N}] \mathcal{P}}^{\mathcal{Q}}+2\left(\hat{\mathcal{L}}_{\Lambda}^{(-1)} \boldsymbol{\Gamma} \cdot \boldsymbol{\Gamma}\right)_{\mathcal{M N \mathcal { N }}}{ }^{\mathcal{Q}} \\
& =\left(\hat{\mathcal{L}}_{\Lambda}^{(-1)} \mathbf{R}\right)_{\mathcal{M N \mathcal { N }}}{ }^{\mathcal{Q}}
\end{aligned}
$$

Therefore, the generalised Riemann tensor transforms as a generalised density of weight -1 .

Open Access. This article is distributed under the terms of the Creative Commons Attribution License (CC-BY 4.0), which permits any use, distribution and reproduction in any medium, provided the original author(s) and source are credited.

\section{References}

[1] H. Godazgar, M. Godazgar and H. Nicolai, Generalised geometry from the ground up, JHEP 02 (2014) 075 [arXiv: 1307.8295] [INSPIRE].

[2] B. de Wit and H. Nicolai, $d=11$ Supergravity with local SU(8) invariance, Nucl. Phys. B 274 (1986) 363 [InSPIRE].

[3] E. Cremmer and B. Julia, The $N=8$ supergravity theory. 1. The Lagrangian, Phys. Lett. B 80 (1978) 48 [INSPIRE].

[4] E. Cremmer and B. Julia, The SO(8) Supergravity, Nucl. Phys. B 159 (1979) 141 [InSPIRE].

[5] E. Cremmer, B. Julia and J. Scherk, Supergravity theory in eleven-dimensions, Phys. Lett. B 76 (1978) 409 [InSPIRE].

[6] H. Godazgar, M. Godazgar and H. Nicolai, Non-linear Kaluza-Klein theory for dual fields, Phys. Rev. D88 (2013) 125002 [arXiv:1309.0266] [inSPIRE]. 
[7] H. Godazgar, M. Godazgar and H. Nicolai, The embedding tensor of Scherk-Schwarz flux compactifications from eleven dimensions, Phys. Rev. D 89 (2014) 045009 [arXiv: 1312.1061] [INSPIRE].

[8] A. Coimbra, C. Strickland-Constable and D. Waldram, $E_{d(d)} \times \mathbb{R}^{+}$generalised geometry, connections and M-theory, JHEP 02 (2014) 054 [arXiv:1112.3989] [INSPIRE].

[9] D.S. Berman, M. Cederwall, A. Kleinschmidt and D.C. Thompson, The gauge structure of generalised diffeomorphisms, JHEP 01 (2013) 064 [arXiv: 1208.5884] [INSPIRE].

[10] O. Hohm and H. Samtleben, Exceptional Field Theory II: $E_{7(7)}$, Phys. Rev. D 89 (2014) 066017 [arXiv:1312.4542] [INSPIRE].

[11] B. de Wit and H. Nicolai, Deformations of gauged $\mathrm{SO}(8)$ supergravity and supergravity in eleven dimensions, JHEP 05 (2013) 077 [arXiv: 1302.6219] [INSPIRE].

[12] D.S. Berman, H. Godazgar, M.J. Perry and P. West, Duality invariant actions and generalised geometry, JHEP 02 (2012) 108 [arXiv:1111.0459] [INSPIRE].

[13] C. Hillmann, Generalized $E_{7(7)}$ coset dynamics and $D=11$ supergravity, JHEP 03 (2009) 135 [arXiv:0901.1581] [INSPIRE].

[14] A.B. Borisov and V.I. Ogievetsky, Theory of dynamical affine and conformal symmetries as gravity theory, Theor. Math. Phys. 21 (1975) 1179 [InSPIRE].

[15] P.C. West, Hidden superconformal symmetry in M-theory, JHEP 08 (2000) 007 [hep-th/0005270] [INSPIRE].

[16] F. Riccioni and P. West, Local E $E_{11}$, JHEP 04 (2009) 051 [arXiv:0902.4678] [InSPIRE].

[17] O. Hohm and H. Samtleben, Exceptional form of $D=11$ supergravity, Phys. Rev. Lett. 111 (2013) 231601 [arXiv:1308.1673] [INSPIRE].

[18] H. Nicolai and H. Samtleben, Maximal gauged supergravity in three-dimensions, Phys. Rev. Lett. 86 (2001) 1686 [hep-th/0010076] [INSPIRE].

[19] H. Nicolai and H. Samtleben, Compact and noncompact gauged maximal supergravities in three-dimensions, JHEP 04 (2001) 022 [hep-th/0103032] [INSPIRE].

[20] B. de Wit, H. Samtleben and M. Trigiante, On Lagrangians and gaugings of maximal supergravities, Nucl. Phys. B 655 (2003) 93 [hep-th/0212239] [INSPIRE].

[21] B. de Wit, H. Samtleben and M. Trigiante, Gauging maximal supergravities, Fortsch. Phys. 52 (2004) 489 [hep-th/0311225] [INSPIRE].

[22] B. de Wit, H. Samtleben and M. Trigiante, The maximal D=4 supergravities, JHEP 06 (2007) 049 [arXiv:0705.2101] [INSPIRE].

[23] B. de Wit, H. Nicolai and N.P. Warner, The embedding of gauged $N=8$ supergravity into $d=11$ supergravity, Nucl. Phys. B 255 (1985) 29 [InSPIRE].

[24] H. Godazgar, M. Godazgar and H. Nicolai, Testing the non-linear flux ansatz for maximal supergravity, Phys. Rev. D 87 (2013) 085038 [arXiv:1303.1013] [INSPIRE].

[25] B. de Wit and H. Nicolai, The consistency of the $S^{7}$ truncation in $D=11$ supergravity, Nucl. Phys. B 281 (1987) 211 [InSPIRE].

[26] H. Nicolai and K. Pilch, Consistent truncation of $D=11$ supergravity on $A d S_{4} \times S^{7}$, JHEP 03 (2012) 099 [arXiv:1112.6131] [INSPIRE]. 
[27] K. Lee, C. Strickland-Constable and D. Waldram, Spheres, generalised parallelisability and consistent truncations, arXiv:1401.3360 [INSPIRE].

[28] C.M. Hull, Generalised geometry for M-theory, JHEP 07 (2007) 079 [hep-th/0701203] [INSPIRE].

[29] P.P. Pacheco and D. Waldram, M-theory, exceptional generalised geometry and superpotentials, JHEP 09 (2008) 123 [arXiv:0804.1362] [INSPIRE].

[30] D.S. Berman and M.J. Perry, Generalized geometry and M-theory, JHEP 06 (2011) 074 [arXiv: 1008.1763] [INSPIRE].

[31] A. Coimbra, C. Strickland-Constable and D. Waldram, Supergravity as generalised geometry II: $E_{d(d)} \times \mathbb{R}^{+}$and M-theory, JHEP 03 (2014) 019 [arXiv:1212.1586] [InSPIRE].

[32] G. Aldazabal, M. Graña, D. Marqués and J.A. Rosabal, Extended geometry and gauged maximal supergravity, JHEP 06 (2013) 046 [arXiv: 1302.5419] [INSPIRE].

[33] M. Cederwall, J. Edlund and A. Karlsson, Exceptional geometry and tensor fields, JHEP 07 (2013) 028 [arXiv: 1302.6736] [INSPIRE].

[34] C. Strickland-Constable, Subsectors, Dynkin diagrams and new generalised geometries, arXiv: 1310.4196 [INSPIRE].

[35] G. Aldazabal, M. Graña, D. Marqués and J.A. Rosabal, The gauge structure of exceptional field theories and the tensor hierarchy, JHEP 04 (2014) 049 [arXiv:1312.4549] [INSPIRE].

[36] E. Cartan, Les groupes des transformations continués, infinis, simples, Ann. Sci. Ecole Norm. Sup. 26 (1909) 93.

[37] I.M. Singer and S. Sternberg, On the infinite groups of Lie and Cartan I, J. Analyse Math. 15 (1965) 1.

[38] V.G. Kac, Classification of infinite dimensional simple groups of supersymmetries and quantum field theory, math/9912235 [INSPIRE].

[39] K. Koepsell, H. Nicolai and H. Samtleben, An exceptional geometry for D $=11$ supergravity?, Class. Quant. Grav. 17 (2000) 3689 [hep-th/0006034] [INSPIRE].

[40] O. Hohm and B. Zwiebach, Large gauge transformations in double field theory, JHEP 02 (2013) 075 [arXiv: 1207.4198] [INSPIRE].

[41] J.-H. Park, Comments on double field theory and diffeomorphisms, JHEP 06 (2013) 098 [arXiv: 1304.5946] [INSPIRE].

[42] D.S. Berman, M. Cederwall and M.J. Perry, Global aspects of double geometry, arXiv: 1401.1311 [INSPIRE].

[43] G. Papadopoulos, Seeking the balance: Patching double and exceptional field theories, arXiv: 1402.2586 [INSPIRE].

[44] B. de Wit and H. Nicolai, Hidden symmetries, central charges and all that, Class. Quant. Grav. 18 (2001) 3095 [hep-th/0011239] [INSPIRE].

[45] M. Graña, R. Minasian, M. Petrini and D. Waldram, T-duality, generalized geometry and non-geometric backgrounds, JHEP 04 (2009) 075 [arXiv:0807.4527] [INSPIRE].

[46] D.S. Berman, H. Godazgar, M. Godazgar and M.J. Perry, The local symmetries of M-theory and their formulation in generalised geometry, JHEP 01 (2012) 012 [arXiv:1110.3930] [INSPIRE]. 
[47] W. Siegel, Superspace duality in low-energy superstrings, Phys. Rev. D 48 (1993) 2826 [hep-th/9305073] [INSPIRE].

[48] O. Hohm, C. Hull and B. Zwiebach, Generalized metric formulation of double field theory, JHEP 08 (2010) 008 [arXiv: 1006.4823] [INSPIRE].

[49] C.D.A. Blair, E. Malek and J.-H. Park, M-theory and Type IIB from a Duality Manifest Action, JHEP 01 (2014) 172 [arXiv:1311.5109] [INSPIRE].

[50] O. Hohm and H. Samtleben, Exceptional field theory I: $E_{6(6)}$ covariant Form of M-theory and type IIB, Phys. Rev. D 89 (2014) 066016 [arXiv:1312.0614] [InSPIRE].

[51] N. Hitchin, Generalized Calabi-Yau manifolds, Quart. J. Math. Oxford Ser. 54 (2003) 281 [math/0209099] [INSPIRE].

[52] M. Gualtieri, Generalized complex geometry, math/0401221 [INSPIRE].

[53] G. Aldazabal, W. Baron, D. Marques and C. Núñez, The effective action of double field theory, JHEP 11 (2011) 052 [Erratum ibid. 1111 (2011) 109] [arXiv:1109.0290] [InSPIRE].

[54] D.S. Berman, E.T. Musaev, D.C. Thompson and D.C. Thompson, Duality invariant M-theory: Gauged supergravities and Scherk-Schwarz reductions, JHEP 10 (2012) 174 [arXiv: 1208.0020] [INSPIRE].

[55] M. Cederwall, J. Edlund and A. Karlsson, Exceptional geometry and tensor fields, JHEP 07 (2013) 028 [arXiv: 1302.6736] [INSPIRE].

[56] F.W. Hehl, P. Von Der Heyde, G.D. Kerlick and J.M. Nester, General relativity with spin and torsion: Foundations and prospects, Rev. Mod. Phys. 48 (1976) 393 [INSPIRE].

[57] J.-H. Park and Y. Suh, U-geometry: SL(5), JHEP 04 (2013) 147 [Erratum ibid. 1311 (2013) 210] [arXiv:1302.1652] [INSPIRE].

[58] I. Jeon, K. Lee and J.-H. Park, Differential geometry with a projection: Application to double field theory, JHEP 04 (2011) 014 [arXiv: 1011.1324] [INSPIRE].

[59] O. Hohm and S.K. Kwak, Frame-like geometry of double field theory, J. Phys. A 44 (2011) 085404 [arXiv:1011.4101] [InSPIRE].

[60] I. Jeon, K. Lee and J.-H. Park, Stringy differential geometry, beyond Riemann, Phys. Rev. D 84 (2011) 044022 [arXiv: 1105.6294] [INSPIRE].

[61] A. Coimbra, C. Strickland-Constable and D. Waldram, Supergravity as generalised geometry I: Type II theories, JHEP 11 (2011) 091 [arXiv:1107.1733] [inSPIRE].

[62] O. Hohm and B. Zwiebach, On the Riemann tensor in double field theory, JHEP 05 (2012) 126 [arXiv:1112.5296] [INSPIRE].

[63] D.S. Berman, C.D.A. Blair, E. Malek and M.J. Perry, The $O_{D, D}$ geometry of string theory, arXiv:1303.6727 [INSPIRE]. 